\title{
Up-regulation of sarcoplasmic reticulum function protects skeletal muscle against cytoplasmic calcium overload during hibernation in ground squirrels
}

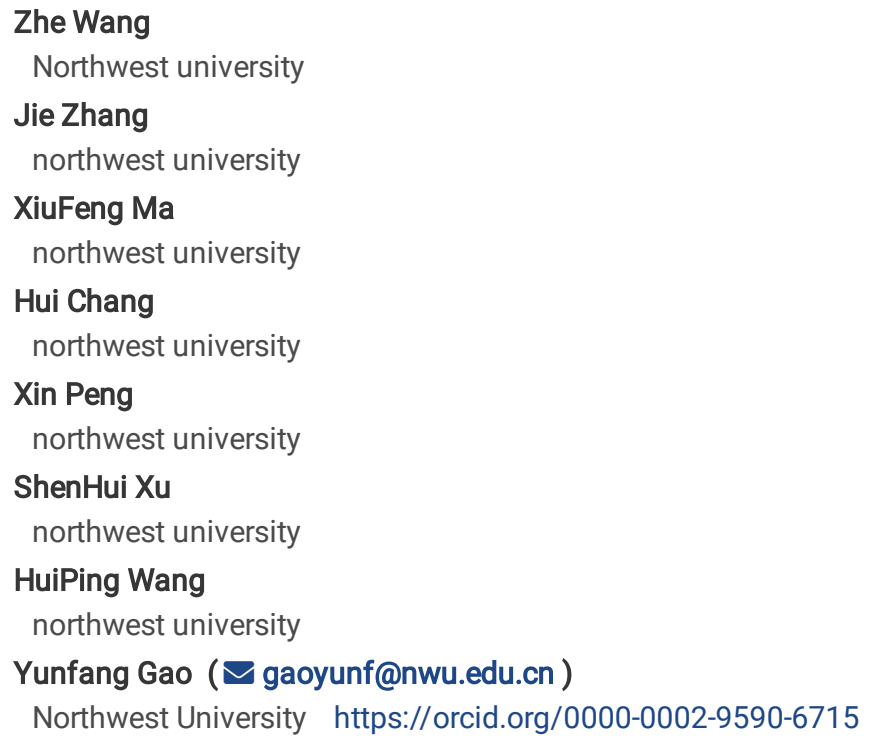

\section{Research}

Keywords: hibernation, calcium homeostasis, skeletal muscle, calcium pump, ryanodine receptor

Posted Date: February 3rd, 2020

DOI: https://doi.org/10.21203/rs.2.22469/v1

License: (c) (i) This work is licensed under a Creative Commons Attribution 4.0 International License. Read Full License 


\section{Abstract}

We investigated the potential mechanism of the (sarcoplasmic reticulum) SR in maintenance of calcium ( $\mathrm{Ca}^{2+}$ ) homeostasis of slow-twitch muscle (soleus, SOL), fast-twitch muscle (extensor digitorum longus, EDL) and mixed muscle (gastrocnemius, GAS) in hibernating ground squirrels (Spermophilus dauricus). Results showed that cytosolic and SR Ca ${ }^{2+}$ concentrations in distinct skeletal muscle fibers increased and decreased during late torpor, respectively, but both returned to summer-active levels during early torpor. Ryanodine receptor1 (RyR1) and sarco/endoplasmic reticulum $\mathrm{Ca}^{2+}$ ATPase isoform 1 (SERCA1) protein expression increased during hibernation. Up-regulation factors of SERCA activity: Phospholamban phosphorylation increased in the SOL and GAS, $\beta$-adrenergic receptor-2 protein expression increased in the GAS, and calmodulin kinase-2 phosphorylation increased in the SOL during hibernation. Down-regulation factors of SERCA activity: Sarcolipin and SERCA1 co-localization decreased in the EDL and GAS. These data suggest that SERCA activity in skeletal muscle fibers increases likely during hibernation. FKBP12/calsequestrin1 (negative regulatory factors of RyR1) and RyR1 co-localization decreased in the GAS, indicating that the RyR1 channel opening probability increased during hibernation. Dihydropyridine receptors protein expression and its co-localization with RYR1 decreased during hibernation prompts that the contractility of skeletal muscle was weakened. Protein expression of $\mathrm{Ca}^{2+}$-binding proteins calsequestrin 1 and calmodulin increased indicating that the ability of intracellular free calcium binding increased during whole hibernation period. These findings confirm that the release, uptake, and binding of free $\mathrm{Ca}^{2+}$ in the SR were enhanced in different skeletal muscles during hibernation. Up-regulation of muscular sarcoplasmic reticulum function protects skeletal muscle fibers against cytoplasmic calcium overload during hibernation in ground squirrels.

\section{Introduction}

Calcium $\left(\mathrm{Ca}^{2+}\right)$ homeostasis is important for maintaining intracellular homeostasis in mammals [1-3]. Under normal conditions, intracellular $\mathrm{Ca}^{2+}$ concentration is accurately and steadily regulated and maintained. However, prolonged skeletal muscle disuse (e.g., during spaceflight, hindlimb unloading, and bed rest) can lead to disturbance of intracellular $\mathrm{Ca}^{2+}$ homeostasis, mainly exhibited by cytoplasmic Ca ${ }^{2+}$ overload [46]. Intracellular $\mathrm{Ca}^{2+}$ overload plays an important role in the mechanisms of disuse-induced muscle atrophy. Increased intracellular Ca ${ }^{2+}$ concentration can activate certain proteases (e.g., calpains), followed by the proteolysis pathway to decompose myofibrillar proteins [4, 7, 8]. Studies have shown that only 2 days (d) of hindlimb immobilization can result in a $38 \%$ increase in cytosolic $\mathrm{Ca}^{2+}$ concentration in the soleus muscle (SOL) of mice, with $\mathrm{Ca}^{2+}$ overload of $117 \%$ reached after $7 \mathrm{~d}$ of disuse [9]. Our previous study also found increases of $330 \%$ and $189 \%$ in cytoplasmic resting $\mathrm{Ca}^{2+}$ concentration in the SOL and gastrocnemius muscle (GAS), respectively, of rats after $14 \mathrm{~d}$ of hindlimb unloading [10, 11]. Cytoplasmic $\mathrm{Ca}^{2+}$ overload and increased protein degradation triggered by the calpain system is considered a major pathway in disuseinduced muscle atrophy $[5,12]$.

Hibernation is an important strategy for survival under low environmental temperatures and food scarcity during winter [13]. Numerous hibernators, including Daurian ground squirrels (Spermophilus dauricus), avoid significant loss of muscle mass and force during prolonged fasting and hibernation inactivity, thus providing a natural model to study the mechanisms involved in the prevention of disuse-induced skeletal muscle atrophy $[7,14,15]$. Our previous study showed the occurrence of intracellular $\mathrm{Ca}^{2+}$ overload during inter-bout arousal in skeletal muscle fibers of ground squirrels, but a reduction in $\mathrm{Ca}^{2+}$ overload after inter-bout arousal; in post-hibernation, the concentration of cytosolic Ca ${ }^{2+}$ in

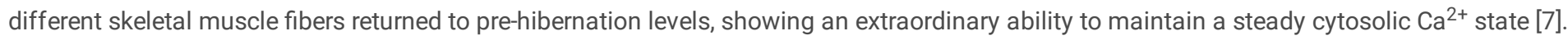
Thus, during long-term hibernation, the torpor-arousal cycle may act to protect skeletal muscles against atrophy by alleviating excessive Ca ${ }^{2+}$ overload in the cytoplasm of muscle fibers and against the resulting increase in protein degradation. Therefore, studies on the mechanisms of $\mathrm{Ca}^{2+}$ homeostasis in the skeletal muscle fibers of hibernating animals are important.

The dynamic balance of $\mathrm{Ca}^{2+}$ between intracellular $\mathrm{Ca}^{2+}$ pools, cytoplasm and extracellular $\mathrm{Ca}^{2+}$ is the main factor affecting the intracellular calcium homeostasis. The endoplasmic reticulum, mitochondria, and nuclei are three $\mathrm{Ca}^{2+}$ pools used in the maintenance of $\mathrm{Ca}^{2+}$ homeostasis

$[16,17]$. The endoplasmic reticulum, called the SR in skeletal muscle, is the most important organelle to maintain intracellular Ca ${ }^{2+}$ homeostasis [18-21]. At present, research on calcium homeostasis in non-hibernating and hibernating animals were mainly focused on SR under the condition of disused skeletal muscle, with few reports on the mitochondria or nuclei [22-28].

Ryanodine receptor (RyR) is a $\mathrm{Ca}^{2+}$-activated $\mathrm{Ca}^{2+}$ channel located on the sarcoplasmic reticulum membrane, that releases $\mathrm{SR}$ Ca ${ }^{2+}$ into the cytoplasm along a concentration gradient when the channels are open [29-32]. Mammalian tissues express RyR1 in skeletal muscle, where the opening of channel is regulated by many factors such as dihydropyridine receptor (DHPR), SR Ca ${ }^{2+}$, calsequestrin1 (CSQ1), and $12-\mathrm{kDa}$ FK506 binding protein (FKBP12) [33]. The structural coupling between DHPR and RyR1 is an important mechanism of $\mathrm{Ca}^{2+}$ release from the SR during muscle contraction [34, 35]. SR free $\mathrm{Ca}^{2+}$ promote the channel opening by combining with RyR1 [36-38]. While, FKBP12 and CSQ1 inhibits the opening of channels by combining with RyR1 in skeletal muscle $[39,40]$. The protein expression of RyR1 is significantly increased in mice with 
GAS atrophy caused by denervation and in rats with SOL atrophy caused by hindlimb unloading [24, 41], the increase of RYR1 expression level and the opening probability may be one of the important mechanisms of calcium overload in skeletal muscle fibers.

The uptake of $\mathrm{Ca}^{2+}$ in the SR depends on the $\mathrm{Ca}^{2+}$ pump (SR Ca ${ }^{2+}$ transport ATPase, SERCA), which transports Ca ${ }^{2+}$ from the cytoplasm to SR along a reverse concentration gradient [42-45]; with SERCA1, followed by SERCA2, mainly expressed in skeletal muscle [46, 47], where their activities are regulated by inhibition of phospholamban (PLB) and sarcolipin (SLN) through combining with SERCA [48-50]. $\beta$-adrenergic receptor2 ( $\beta$-AR2) located on the cell membrane can increasing SERCA activity through cascade amplification of intracellular cAMP-PKA signaling [51]. Cytoplasmic calmodulin kinase2 (CaMK2) also can enhance SERCA activity by its own phosphorylation [52, 53]. Research has shown that SERCA1 protein expression and $\mathrm{Ca}^{2+}$ pump activity decreases $\mathrm{Ca}^{2+}$ in SOL of hindlimb-unloading rats [22,54]. The decrease of SR $\mathrm{Ca}^{2+}$ uptake may be one of the causes of $\mathrm{Ca}^{2+}$ overload in atrophic skeletal muscle and the regulation of protein expression and activity of calcium pump is the core mechanism of skeletal muscle fiber to inhibit cytoplasmic $\mathrm{Ca}^{2+}$ overload.

In addition to the regulation of $\mathrm{SR}$, intracellular $\mathrm{Ca}^{2+}$ homeostasis also depends on the dynamic balance between free and bound $\mathrm{Ca}^{2+}$. Under normal conditions, more than $90 \%$ of $\mathrm{Ca}^{2+}$ is stored for buffering in the form of bound $\mathrm{Ca}^{2+}[55]$. Calmodulin (CaM) is a Ca ${ }^{2+}$-binding protein located in the cytoplasm and can directly reduce the concentration of cytosolic free $\mathrm{Ca}^{2+}$ by combining with four Ca ${ }^{2+}[56,57]$. CSQ1 is the most abundant $\mathrm{Ca}^{2+}$-binding protein in the SR of skeletal and cardiac muscle; furthermore, each CSQ1 molecule can combine with 43 Ca ${ }^{2+}$, thereby greatly reducing the concentration of free $\mathrm{Ca}^{2+}$ in the SR $[38,55,58,59]$. Protein expression of CSQ1 increases significantly in the SOL and lateral femoral muscles of rats after $7 \mathrm{~d}$ of hindlimb disuse prompting that the disuse of skeletal muscle may also cause changes in the expression of calcium binding protein in muscle fibers $[22,25]$. These two $\mathrm{Ca}^{2+}$ binding proteins reduce the free $\mathrm{Ca}^{2+}$ concentration in the cytoplasm or SR by binding free $\mathrm{Ca}^{2+}$, which is involved in the maintenance of calcium homeostasis in skeletal muscle fibers.

In brief, previous studies have demonstrated that the increase in RyR1 protein expression and decrease in SERCA protein expression are important mechanisms that can lead to $\mathrm{Ca}^{2+}$ overload and atrophy in skeletal muscles of non-hibernating animals. Compared with nonhibernating animals, there were few studies on $\mathrm{SR} \mathrm{Ca}^{2+}$ related protein in hibernating animals, and the results were different. Research on mixed skeletal muscles in the hindlimbs of Siberian ground squirrels (Spermophilus undulatus) reported significant decreases in the protein expression levels of SERCA1, RyR1, and CSQ1 during hibernation [27]. Conversely, we previously showed that Ca ${ }^{2+}$ pump (SERCA1 and SERCA2) activity increases significantly in the SOL and EDL muscles of ground squirrels during hibernation and inter-bout arousal [60], CaM protein levels increase significantly in mixed hindlimb skeletal muscles of hibernating thirteen-lined ground squirrels (Ictidomys tridecemlineatus) [28]. More importantly, the underlying mechanisms of these changes, especially the regulation of RYR1 and calcium pump activity, is not clear. Thus, it is necessary to systematically study the expression and regulation of SR $\mathrm{Ca}^{2+}$-related proteins in different stages of hibernation to clarify the occurrence of cytosolic $\mathrm{Ca}^{2+}$ overload in some stages of hibernation and the mechanism of partial or total recovery of $\mathrm{Ca}^{2+}$ homeostasis in skeletal muscle fibers after interbout arousal.

In addition, our previous research showed that the response of different muscle types to hibernation appears to be related to fiber type. For example, post inter-bout arousal, cytoplasmic $\mathrm{Ca}^{2+}$ concentrations recover to pre-hibernation levels in the SOL and EDL, but remain higher in the GAS in the same period [7]. We hypothesize that hibernating animals maintain the dynamic balance between SR and cytoplasmic Ca ${ }^{2+}$ by regulating $\mathrm{Ca}^{2+}$ uptake and excretion channels of the $\mathrm{SR}$, which is an important mechanism to avoid or reduce cytoplasmic $\mathrm{Ca}^{2+}$ overload, with this regulatory mechanism possibly related to muscle fiber type. To test this hypothesis, we used slow SOL, fast EDL, and mixed GAS muscles of Daurian ground squirrels to study cytosolic and $\mathrm{SR} \mathrm{Ca}^{2+}$ concentrations in skeletal muscle fibers, as well as the protein and mRNA expression levels of SERCA and RyR signaling pathways (including SERCA1, PLB, SLN, $\beta$-AR2, CaMK2, RyR1, DHPR, and FKBP12), co-localization between SERCA and RyR1 with their distinct proteins, and expression levels of $\mathrm{Ca}^{2+}$-binding proteins (CSQ1, CaM). We further explored the molecular mechanism of $\mathrm{SR}$ involvement in the regulation of $\mathrm{Ca}^{2+}$ homeostasis in mammalian hibernators.

\section{Methods}

Ethical approval. All procedures were approved by the Laboratory Animal Care Committee of the China Ministry of Health. The Northwest University Ethics Committee reviewed and approved all animal study procedures. All procedures were carried out in accordance with approved guidelines.

Animals and groups. Daurian ground squirrels (Spermophilus dauricus) were prepared as described in our previous work [14, 61]. Briefly, ground squirrels (male to female ratio of 1:2) were caught within the Weinan region at May each year in Shaanxi Province, China, and then transferred to our laboratory. The ground squirrels were individually housed under normal laboratory illumination and temperature conditions in $50 \times 50 \times$ $20 \mathrm{~cm}$ cages and were provided with water and rat chow ad libitum. The animals were acclimated for one week before experiments commenced. A total of 96 adult active and healthy ground squirrels (300-400 g) were weighed and selected. When the squirrels entered torpor (early November), they were transferred to a cold room $\left(4-6^{\circ} \mathrm{C}\right)$. The commencement of torpor and torpor dates were determined by placing sawdust

Page $3 / 24$ 
on the back of each subject (to observe movement) and when body temperature $\left(T_{b}\right)$ fell below $9{ }^{\circ} \mathrm{C}$, as determined by thermal visual imaging (Fluke VT04 Visual IR Thermometer, USA). Once squirrels entered torpor, food and water were removed and daily observations were conducted for the entire experimental period. Based on our annual records, squirrels resume hibernation after short periods (1-2 d) of inter-bout arousal. Animals that aroused for more than $2 \mathrm{~d}$ (without re-entering hibernation) were assigned to the post-hibernation group.

After matching for body mass, animals were randomly assigned to six groups ( $n=16)$ : (1) summer active (SA): before July and maintaining a $T_{b}$ of $36-38^{\circ} \mathrm{C}$, representing the control group during different hibernation periods and the most active state; (2) pre-hibernation (PRE): nonhibernating animals in late-autumn and maintaining a $\mathrm{T}_{\mathrm{b}}$ of $36-38^{\circ} \mathrm{C}$, representing the hibernating control group and the pre-fattening state; (3) late torpor (LT): after two months of hibernation, with animals maintaining a $\mathrm{T}_{\mathrm{b}}$ of $5-8{ }^{\circ} \mathrm{C}$ more than $5 \mathrm{~d}$; (4) interbout arousal (IBA): after two months of hibernation, awake animals with $\mathrm{T}_{\mathrm{b}}$ returned to $34-37^{\circ} \mathrm{C}$ for less than $12 \mathrm{~h}$; (5) early torpor (ET): after two months hibernation, animals entered into a new hibernation bout with Tb maintained at $5-8{ }^{\circ} \mathrm{C}$ for less than $24 \mathrm{~h}$ (the LT, IBA, and ET three groups together constitute the hibernation-awakening cycle, representing three different hibernation states before, during, and after inter-bout arousal); (6) post-hibernation (POST): animals awaking from hibernation and maintaining a $T_{b}$ of $36-38{ }^{\circ} \mathrm{C}$ for more than $2 \mathrm{~d}$ in March of the following year, representing the degree of body state recovery of dormant ground squirrels.

Isolation of single muscle fibers. Animals were deeply anaesthetized with sodium pentobarbital (90 mg/kg). Muscle samples with tendons were dissected carefully from surrounding tissues and sarcolemma, ensuring intact nerves and blood supply. The muscles were separated into two complete strips along the longitudinal axis using tweezers, then rinsed with $20 \mathrm{~mL}$ of phosphate-buffered saline (PBS, $137 \mathrm{mM}$ sodium chloride, $4.3 \mathrm{mM}$ disodium chloride, $2.7 \mathrm{mM}$ potassium chloride, $1.4 \mathrm{mM}$ monopotassium phosphate, $\mathrm{pH}$ 7.4), acutely dissociated with $3 \mathrm{~mL}$ of enzymatic digestion solution consisting of $0.35 \%$ collagenase I and $0.17 \%$ neutral protease (Sigma-Aldrich, Saint Quentin Fallavier, France), and finally incubated at $33{ }^{\circ} \mathrm{C}$ on an orbital shaker for $2 \mathrm{~h}$. The enzymatic digestion solution was saturated with $95 \% \mathrm{O}_{2}$ and $5 \% \mathrm{CO}_{2}$ gas mixture to ensure the muscle fibers were completely digested, after which the solution was removed with PBS and the muscles were agitated gently and repeatedly with pipettes [62]. The dissociated single muscle fibers were set onto culture chamber slides and finally observed under an inverted microscope (Olympus, IX2-ILL100, Japan).

Muscle samples for other experiments were subsequently stored in liquid nitrogen until further processing. At the end of surgical intervention, the animals were sacrificed by an overdose injection of sodium pentobarbital. The Northwest University Ethics Committee reviewed and approved all animal study procedures. All procedures were carried out in accordance with approved guidelines.

Measurement of cytoplasm $\mathrm{Ca}^{2+}$. Fluo-3-acetoxymethylester (Fluo-3/AM) (Invitrogen, Carlsbad, USA), which exhibits an increase in fluorescence upon $\mathrm{Ca}^{2+}$ binding, was used to measure cytosolic free $\mathrm{Ca}^{2+}$, as described previously [63]. In brief, the above isolated muscle fibers were incubated in glass petri dishes with Fluo-3/AM at a concentration of $5 \mathrm{mM}$ for $30 \mathrm{~min}$ at $37^{\circ} \mathrm{C}$, after which the Fluo-3/AM-loaded muscle fibers were washed with fresh PBS and then scanned under a laser confocal microscope equipped with the Olympus FV10-ASW system (krypton/argon laser illumination at $488 \mathrm{~nm}$ and capture at $526 \mathrm{~nm}$ ). A single muscle fiber with intact morphology and smooth cytomembrane was found at low magnification (100.), with continuous photographs taken of the middle two-thirds segment of the selected muscle fiber at high magnification (400·). Six different areas were randomly selected for fluorescence intensity measurements in each image. Total fluorescence intensity / total area of the selected region was used as the average fluorescence intensity of the muscle fiber, which represented the concentration of $\mathrm{Ca}^{2+}$ labeled. The average value of the measured result was taken as the fluorescence intensity of the muscle fiber cytosolic $\mathrm{Ca}^{2+}$ concentration. The

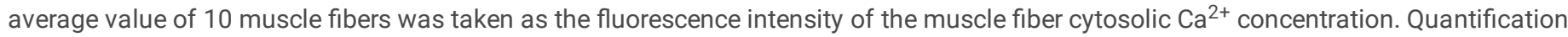
analysis of the fluorescence intensity was performed with $\mathrm{NIH}$ Image $\mathrm{J}$ software (Image-ProPlus 6.0).

Measurement of SR Ca ${ }^{2+}$. Magnesium-Fluo-4-acetoxymethylester (mag-Fluo-4/AM) (\#M14206, Thermo Fisher Scientific, Rockford, IL, USA), which exhibits an increase in fluorescence upon binding to $\mathrm{Ca}^{2+}$, was used to indicate $\mathrm{SR}$ free $\mathrm{Ca}^{2+}$, as described previously [64]. Briefly, single muscle fibers were incubated with mag-Fluo-4/AM (5 mM) and ER-Tracker Red dye (\#E34250, Thermo Fisher Scientific) for 30 min at $37{ }^{\circ} \mathrm{C}$. After incubation on glass petri dishes, the mag-Fluo-4/AM-loaded muscle fibers were washed with fresh PBS and then scanned under a laser confocal microscope equipped with the Olympus FV10-ASW system (Olympus, FV10-MCPSU, Japan) with krypton/argon laser illumination at 488 nm and capture at $526 \mathrm{~nm}$. Average fluorescence intensity was used to indicate changes in SR Ca ${ }^{2+}$ in muscle fibers, with the specific method similar to measurement of cytoplasm $\mathrm{Ca}^{2+}$. Quantification analysis of fluorescence intensity was performed with $\mathrm{NIH} \mathrm{Image} \mathrm{J}$ software.

Co-localization analysis of immunohistochemistry. We cut 10- $\mu$ m thick frozen muscle cross-sections from the mid-belly of each muscle at $20^{\circ} \mathrm{C}$ with a cryostat (Leica, Wetzlar, CM1850, Germany), which were then stored at $-80^{\circ} \mathrm{C}$ for further staining. Immunohistochemistry was used to determine co-localization with DHPR/RyR1, CSQ1/RyR1, FKBP12/RyR1, SLN/SERCA1, and SLN/SERCA2. After air drying for 2 h, the sections were incubated in a blocking solution (5\% BSA) (Boster, Wuhan, China) for 10 min at room temperature and, in turn, incubated in a primary antibody (Table 1) solution at $4{ }^{\circ} \mathrm{C}$ overnight. On the following day, the sections were incubated with secondary antibody at $37{ }^{\circ} \mathrm{C}$ for $2 \mathrm{~h}$. After this, the sections were incubated with another primary antibody and secondary antibody under the same conditions. The details of primary and secondary antibodies are listed in Table 2. Finally, the glass slides were placed in 4'-6'-diamidino-2-phenylindole (DAPI)(1:100, \# D9542, Sigma-

Page $4 / 24$ 
Aldrich) at $37^{\circ} \mathrm{C}$ for $30 \mathrm{~min}$. Images were visualized using a confocal laser scanning microscope by krypton/argon laser illumination at $350 \mathrm{~nm}$, $488 \mathrm{~nm}$, and $647 \mathrm{~nm}$ emitted light, and capture at $461 \mathrm{~nm}, 526 \mathrm{~nm}$, and $665 \mathrm{~nm}$. Six figures were analyzed in each sample and eight samples were analyzed in each group. Pearson coefficient was used to measure the overlap level of two proteins [65], NIH Image software (Image-Proplus 6.0) was used to quantify the co-localization coefficient.

Quantitative real-time PCR. Total RNA was routinely extracted from muscles using an RNAiso Plus kit (TaKaRa, Dalian, China) according to the manufacturer's protocols. We determined RNA quality via the OD260/OD280 ratio; only samples with a ratio $>1.8$ were reverse transcribed into cDNA using a TAKARA reagent (TaKaRa), then stored at $-20^{\circ} \mathrm{C}$ for subsequent reactions. Quantitative real-time PCR (RT-PCR) was performed using a SYBR Premix Ex Taq II kit (TaKaRa). Amplification and dissolution curves were first observed, with the right curve then chosen. Here, atubulin (reference gene) and $2^{-\triangle \Delta c t}$ were used to analyze the relative concentrations of serca1, serca2, sln, plb, csq1, cam, fkbp12, and ryr1 mRNA. The primers used for RT-PCR included (Sangon, Nanjing, China):

serca1 forward: 5'- GGTACTGGTCGGGATACCCT-3',

reverse: 5'- GCTGGATAGAGCCTGTGACC-3',

serca2 forward: 5'- TACTGACCCTGTCCCTGACC-3',

reverse: 5'- CACCACCACTCCCATAGCTT-3',

sin forward: 5'- TCCTGATTACGGTCCTCCTC-3',

reverse: 5'- GCGCTGGCATTATTTAGGAG-3',

plb forward: 5'- CCAATATCTCACTCGCTCTGC-3',

reverse: 5'- ACGATGATGCAAATCAGCAA-3',

csq1 forward: 5'- ATCCCAGACAAACCCAACAG-3',

reverse: 5'- TTCCATCCAGGTCATCTTCC-3',

cam forward: 5'- GGCACCATTGACTTCCCAGA-3',

reverse: 5'- TCTGCCGCACTGATGTAACC-3',

ryr1 forward: 5'-ACCAACTGTGCCCTCTTCTC-3',

reverse: 5'- GACCTCGGGACTCTCAATCA-3',

fkbp12 forward: 5'- GCAGGTGGAGACCATCTCTT-3',

reverse: 5'- TGTCCCGAGAGGAGTCAAAT-3',

a-tubulin forward: 5'- CCTGCTGATGGAACGTCTCT-3',

reverse: 5'- GTAAGCTGTTCATGGTAGGC-3',

Cytosolic protein extraction. A Nuclear/Cytosol Fractionation Kit was used to extract cytoplasmic protein (Biovision, \#K266-25, Mountain View, USA). Cells were collected via centrifugation at $600 \times \mathrm{g}$ for $5 \mathrm{~min}$ at $4{ }^{\circ} \mathrm{C}$. Cytosol Extraction Buffer-A Mix ( $\left.0.2 \mathrm{ml}\right)$ containing DTT and protease inhibitors was added. The tissue samples (100-200 mg) were cut into small pieces and then added to ice cold PBS (1-2 ml) and homogenized in a tissue homogenizer. The cells were pelleted by centrifugation at $500 \times \mathrm{g}$ for $2-3 \mathrm{~min}$ at $4{ }^{\circ} \mathrm{C}$, with the supernatant then removed. We next added $0.2 \mathrm{ml}$ of the Cytosol Extraction Buffer-A mix, followed by vigorous vortexing at the highest setting for $15 \mathrm{~s}$ to fully resuspend the cell pellet. After incubating the tube on ice for $10 \mathrm{~min}, 11 \mu \mathrm{l}$ of ice-cold Cytosol Extraction Buffer-B was added to the tube, followed by vortexing at the highest setting for $5 \mathrm{~s}$, incubation on ice for $1 \mathrm{~min}$, and vortexing at the highest setting for $5 \mathrm{~s}$. After that, the tube was centrifuged at maximal speed for 5 min with a micro centrifuge at $4^{\circ} \mathrm{C}$ and $16,000 \times \mathrm{g}$. Finally, the supernatant (cytoplasmic extract) fraction was transferred to a clean pre-chilled tube immediately.

Western blots. Total protein was extracted from the SOL, EDL, and GAS muscles and solubilized in sample buffer (100 mM Tris, pH 6.8, $5 \% 2-\beta$ mercaptoethanol, $5 \%$ glycerol, $4 \%$ SDS, and bromophenol blue), with the extracts of muscle protein then resolved via SDS-PAGE (10\% Laemmli gel with an acrylamide/bisacrylamide ratio of 29:1 and 98\% 2,2,2-trichloroethanol (Aladdin, JI522028, China). For electrophoresis, two identical plates of gel for sample sequence and protein quantity were used. One plate was used for total protein quantification as an internal reference, 
and the other plate was used for follow-up target protein quantification. After electrophoresis, the proteins were electrically transferred to PVDF membranes (0.45- $\mu \mathrm{m}$ pore size) using a Bio-Rad semi-dry transfer apparatus. The blotted membranes were blocked with $1 \%$ BSA in Tris-buffered saline (TBS; 150 mM NaCl, 50 mM Tris-HCl, pH 7.5) and incubated with rabbit anti-phospholamban (1:1000, \#8495, CST), rabbit anti-phosphophospholamban (1:1000, \#8496, CST), rabbit anti-SERCA1 (1:1000, \#4219, CST), rabbit anti-sarcolipin (1:200, \#18395-1-AP, Proteintech), rabbit anti-calsequestrin 1 (1:10000, \# ab191564, Abcam), rabbit anti-calmodulin 1 (1:1000, \#4830,CST), rabbit anti- $\beta$-adrenergic receptor 2(1:500, \#ab182136, Abcam), rabbit anti-CaMK2 (1:500, \#3357, CST), rabbit anti-phospho-CaMK2 (1:1000, \#12716S, CST), mouse anti-FKBP12 (1:1000, ab58072, Abcam), mouse anti-DHPR (1:1000, SC-21781, Santa Cruz), and rabbit anti-RyR1 (1:1000, \#8153, CST) in TBS containing 0.1\% BSA at $4{ }^{\circ} \mathrm{C}$ overnight. The membranes were then incubated with Goat anti-Rabbit IgG $(\mathrm{H}+\mathrm{L})$ Secondary Antibody (1:5000, \#31460, Thermo Fisher) or Goat anti-Mouse IgG (H+L) Secondary Antibody (1:5000, \#62-6520, Thermo Fisher) for 90 min at room temperature, covered with West Pico PLUS Chemiluminescent Substrate (34580, Thermo Fisher), and visualized with an Odyssey scanner (LI-COR Biosciences, Lincoln, NE, USA) [61]. Quantification analysis of the blots was performed using NIH Image J software. Immunoblot band density in each individual lane was standardized against the summed densities from a group of total protein.

Statistical analyses. One-way ANOVA with Fisher's LSD post hoc test was used to determine group differences, and ANOVA-Dunnett's T3 test was used when no homogeneity was detected. SPSS 19.0 was used for all statistical tests. Statistical significance was accepted at $P<0.05$.

\section{Results}

Changes of body surface temperature of ground squirrels during hibernation. The hibernating ground squirrels are in the cycle of torpor - arousal when they enter hibernation. The body temperature is close to the ambient temperature in torpor. The temperature rises to above $35^{\circ} \mathrm{C}$ in interbout arousal (Fig. 1).

Body weight, skeletal muscle wet weight (MWW), and ratio of skeletal muscle wet weight to body weight (MWW/BW). Three different muscles (SOL, GAS, and EDL) from Daurian ground squirrels were used in the current research. Compared with the summer group (SA), the MWW of the SOL was $14-24 \%(P<0.05)$ lower in the pre-hibernation (PRE), inter-bout arousal (IBA), early torpor (ET), later torpor (LT), and post-hibernation (POST) groups and the MWW of the EDL was $16-21 \%(P<0.05)$ lower in the IBA, ET, LT, and POST groups. Compared with the SA and PRE groups, the MWW of the GAS was 14-25\% ( $<<0.05)$ lower in the IBA, ET, LT, and POST groups (Table 1). Moreover, the MWW/BW ratio in the SOL was $20 \%(P<0.05)$ lower in the PRE and 12-22\% $(P<0.05)$ higher in the other four groups compared with the SA group. The MWW/BW ratio in the GAS and EDL muscles was $14-36 \%(P<0.05)$ higher in the other four groups compared with the SA and PRE groups $($ Table 2$)$.

Cytoplasm $\mathrm{Ca}^{2+}$ concentration in single muscle fiber. Figure 2a shows representative two-dimensional confocal images of muscle fibers from the SOL, GAS, and EDL of the SA, PRE, IBA, ET, LT, and POST groups. The uniform green fluorescence mainly distributed in the cytoplasm represents $\mathrm{Ca}^{2+}$ concentration in the muscle fiber. The cytosolic $\mathrm{Ca}^{2+}$ fluorescence in the GAS increased by $46 \%$ in the ET group compared with that in the SA group. It is worth noting that, compared with the SA group, the $\mathrm{Ca}^{2+}$ fluorescence in the LT and IBA groups increased significantly by $117 \%(P<0.001)$ and $30 \%(P<0.05)$ in the SOL, by 106\% $(P<0.001)$ and 65\% $(P<0.01)$ in the EDL, and by $213 \%(P<0.001)$ and $153 \%(P<$ $0.001)$ in the GAS, respectively. However, compared to the LT group, the $\mathrm{Ca}^{2+}$ fluorescence in the POST group decreased significantly by $56 \%(P<$ $0.001)$ in the SOL and 64\% $(\mathrm{P}<0.001)$ in the GAS. Compared with the ET group, $\mathrm{Ca}^{2+}$ fluorescence in the POST group decreased significantly $(23 \%, \mathrm{P}<0.05)$ in the GAS but increased significantly $(38 \%, \mathrm{P}<0.05)$ in the EDL (Fig. $2 \mathrm{~b})$.

SR-Ca ${ }^{2+}$ concentration in single muscle fiber. Figure 3a shows typical two-dimensional confocal images of muscle fibers showing SR-specific fluorescence and SR-Ca ${ }^{2+}$ fluorescence, respectively. The SR-Ca ${ }^{2+}$ fluorescence almost coincided with the SR fluorescence in muscle fibers and was distributed uniformly along the stria of muscle fibers.

Figure $3 b$ shows representative two-dimensional confocal images of muscle fibers from all three muscles in the SA, PRE, LT, IBA, ET, and POST groups. The muscle fibers exhibited a relatively uniform green fluorescence, distributed mainly in the SR. The SR Ca ${ }^{2+}$ fluorescence in the SOL, EDL, and GAS muscles decreased significantly by $80 \%, 84 \%$, and $68 \%(P<0.001)$, respectively, in the PRE group compared with the SA group. The $\mathrm{Ca}^{2+}$ fluorescence in the SOL showed a slight decrease $(14 \%, P<0.05)$ in the IBA group compared with the SA group. An extremely significant decrease in $\mathrm{Ca}^{2+}$ fluorescence was observed in the EDL $(83 \%, P<0.001)$ and GAS muscles $(65 \%, P<0.001)$. The SR Ca ${ }^{2+}$ fluorescence in the EDL increased by $23 \%(P<0.01)$ in the ET group compared with the SA group; however, no significant differences were observed in the SOL and GAS. The SR Ca ${ }^{2+}$ fluorescence in the SOL and GAS muscles from the LT group decreased and recovered to PRE group levels. The greatest differences among the three different muscles were in the POST group. Compared with the SA group, SR $\mathrm{Ca}^{2+}$ fluorescence in the POST group decreased significantly in the SOL $(18 \%, P<0.05)$ and EDL $(43 \%, P<0.05)$, but increased significantly in the GAS $(40 \%, P<0.05)($ Figure $3 \mathrm{c})$.

Relative protein expression. The protein contents of RyR1, DHPR, FKBP12, SERCA1, SLN, P-PLB, PLB, $\beta$-AR2, P-CaMK2, CaMK2, CaM and CSQ1 were detected by Western blot analysis, as shown in Figure 4a. Representative polyacrylamide gels of total protein are shown in Figure 4b. The protein expression levels of RyR1 in the three muscles in the LT, IBA, and ET groups were higher than levels in the other groups. The increments

Page 6/24 
were $37 \%-51 \%(P<0.01)$ in the SOL, 43\%-50\% in the EDL $(P<0.05)$, and $96 \%-107 \%(P<0.01)$ in the GAS compared to the SA group; however, no significant differences were observed among the three groups (Figure $4 \mathrm{c}$ ).

The protein expression levels of DHPR in the three muscles in the LT, IBA, and ET groups were lower than the levels in the SA group, with decrements of $30 \%-32 \%(P<0.05)$ in the SOL, 33\%-40\% $(P<0.05)$ in the EDL, and $23 \%-29 \%(P<0.05)$ in the GAS (Figure $4 \mathrm{~d})$.

Compared to the SA group, FKBP12 protein expression in the SOL increased by $103 \%$ in the PRE group $(P<0.01)$, 82\% in the LT group $(P<0.01)$, and $52 \%$ in the POST group $(P<0.05)$. Compared to the SA group, FKBP12 protein expression in the EDL increased by $22 \%$ in the PRE $(P<0.05)$ and $50 \%$ in the LT group $(P<0.01)$ but decreased by $22 \%$ in the ET $(P<0.05)$. Compared to the SA group, FKBP12 protein expression in the GAS decreased by $23 \%$ in the PRE $(P<0.05), 18 \%$ in the ET $(P<0.05)$, and $38 \%$ in the LT $(P<0.01)$ (Figure 4e). Overall, the RyR1 regulation pathway was mainly up-regulated during hibernation, except for DHPR.

Compared with the SA group, the SERCA1 protein level increased significantly in the three different muscles by $43 \%-98 \%$ in the IBA group $(P<$ $0.001), 28 \%-103 \%$ in the ET group $(P<0.05)$, and $9 \%-102 \%$ in the LT group $(P<0.05)$, respectively. In the SOL muscle, expression of SERCA1 only decreased $(24 \%, P<0.05)$ in the POST group compared with the SA group. The main difference between the GAS and other two muscles was that a significant increase in SERCA1 expression was observed in both the PRE group $(96 \%, P<0.01)$ and POST group $(145 \%, P<0.001)$ (Figure 4f) compared with the SA group.

The SLN protein expression in the three different muscles showed a common pattern. The protein expression levels in most hibernating groups (PRE, LT, IBA, ET) were not lower than that in the SA group. In the SOL, the increments were $140 \%(P<0.001)$ in the PRE, 136\% $(P<0.001)$ in the LT, and $89 \%(P<0.05)$ in the IBA. In the EDL, the increments were $216 \%(P<0.001)$ in the PRE, $74 \%(P<0.05)$ in the LT, 168\% $(P<0.001)$ in the IBA, and $100 \%(P<0.05)$ in the ET. In the GAS, the increments were $175 \%(P<0.001)$ in the PRE, 53\% $(P<0.05)$ in the LT, and 51\% $(P<0.05)$ in the IBA compared to the SA group, respectively (Figure $4 \mathrm{~g}$ ).

The PLB protein expression in the SOL was $19 \%$ and $21 \%(P<0.05)$ higher in the PRE and ET groups than that in the SA group. Expression in the EDL increased significantly by $27 \%(P<0.05)$ in the PRE group and $24 \%(P<0.05)$ in the LT group but decreased by $21 \%(P<0.05)$ in the POST group. Expression in the GAS was higher in all groups compared with that in the SA group, with increases of $34 \%(P<0.05)$ in the PRE, $117 \%(P<$ $0.001)$ in the LT, $172 \%(P<0.001)$ in the IBA, 112\% $(P<0.001)$ in the ET, and $72 \%(P<0.05)$ in the POST (Figure $4 \mathrm{~h})$.

The $\mathrm{p}-\mathrm{PLB} / \mathrm{PLB}$ ratio, as an important indicator of $\mathrm{Ca}^{2+}$ pump activities, was analyzed in the SOL, EDL, and GAS muscles. In the SOL muscle, the ratio remained unchanged in all hibernation groups, except the IBA group, which was higher $(101 \%, P<0.001)$ than that in the SA group. In the $\mathrm{EDL}$, the ratio was significantly decreased in the LT group $(43 \%, P<0.01)$ compared with the SA group. In the GAS muscle, the ratio was significantly elevated in the IBA $(29 \%, P<0.05)$, ET $(50 \%, P<0.01)$, and POST groups $(30 \%, P<0.05)$ compared with that in the SA group $($ Figure $4 i)$.

The $\beta$-AR2 protein expression in the SOL remained unchanged in all groups, except for the POST group (-72\% compared to SA, $P<0.001)$. In the EDL, protein expression in all groups was significantly lower than that in the SA group $(P<0.05)$. In the GAS, however, protein expression was significantly increased in the PRE $(45 \%, P<0.05)$, IBA $(42 \%, P<0.05)$, and ET groups $(50 \%, P<0.05)$ compared with the SA group (Figure $4 \mathrm{j})$.

Similar CaMK2 protein expression trends were found in the SOL and EDL, with levels in the PRE, IBA, ET, and LT groups higher than that in the SA group. In the SOL and EDL, protein expression increased significantly in the PRE, IBA, ET, and LT groups (33\% and $20 \%, 22 \%$ and $38 \%, 31 \%$ and $34 \%$, and $38 \%$ and $40 \%(P<0.01)$, respectively compared with the SA group. In the GAS, protein expression decreased significantly by $47 \%$ in the LT group $(P<0.01)$ and $33 \%$ in the ET group $(P<0.05)$ compared with the SA group (Figure 4k).

In the SOL, the P-CaMK2 to CaMK2 ratio only increased by $18 \%(P<0.05)$ in IBA group in SOL, while other groups were all lower than that in SA group in three muscles (Figure 4I). Overall, the SERCA regulation pathway was mainly up-regulated during hibernation.

The CaM protein expression showed similar alternation trends in the EDL and GAS muscles, with the level of CaM in the other five groups higher than that in the SA group. In the EDL muscle, the CaM protein expression levels in the PRE, IBA, ET, LT, and POST groups were 48\% ( $P<0.05)$, $126 \%, 124 \%, 125 \%$, and $134 \%(P<0.001)$ higher, respectively, than that in the SA group. In the GAS, protein expression significantly increased by $116 \%, 89 \%, 87 \%, 87 \%(P<0.05)$, and 136\% $(P<0.001)$ in the PRE, IBA, ET, LT, and POST groups, respectively, compared with the SA group. In the SOL, however, protein expression decreased significantly by $75 \%$ and $73 \%(P<0.001)$ in the LT and POST groups, respectively, compared with the SA group (Figure $4 \mathrm{~m}$ ).

Similar change trends in CSQ1 protein expression were observed in the three different muscles, with levels in the five groups higher than that in the SA group. In the SOL, compared with the SA group, protein expression increased significantly by $205 \%(P<0.05)$ in the PRE, 323\% $(P<0.001)$ in the IBA, 334\% $(P<0.001)$ in the ET, 185\% $(P<0.001)$ in the LT, and 114\% $(P<0.05)$ in the POST. In the EDL, protein expression increased significantly by $85 \%$ in the PRE, $81 \%$ in the IBA, $83 \%$ in the ET, and $103 \%$ in the LT $(P<0.05)$. In the GAS, protein expression increased significantly 
by $97 \%$ in the PRE, $280 \%$ in the IBA, $240 \%$ in the ET, $235 \%$ in the LT, and $242 \%$ in the POST $(P<0.05)$ (Figure $4 \mathrm{n})$. Overall, the expression of both proteins showed an increasing trend during hibernation compared to the SA.

Relative mRNA expression. Relative expression levels of serca1, serca2, plb, and s/n are shown in Figures 5a, b, c, and d, respectively. Commonly, the mRNA expression of serca 1 in the different muscles was lower in the POST group than in the PRE group. In the SOL, the expression levels in the PRE $(38 \%, P<0.01)$, IBA $(63, P<0.001)$, and POST groups $(47 \%, P<0.001)$ were significantly lower than that in the SA group. In the EDL, expression levels in the PRE and POST groups were $23 \%$ and $34 \%(P<0.05)$ lower and in the ET group was $21 \%(P<0.05)$ higher than that in the SA group (Figure 5a).

Compared with the SA group, the relative serca2 mRNA expression in most groups increased in the three different muscles. In the SOL, expression increased significantly by $95 \%-313 \%(P<0.05)$ in the PRE, IBA, ET, and LT groups. In the EDL, expression increased significantly by $500 \%-900 \%(P<0.001)$ in the PRE, IBA, and ET groups. In the GAS, expression increased significantly by $100 \%-176 \%$ in all groups, except the POST group (Figure 5b).

In the SOL, compared with the SA group, the relative $p / b$ mRNA expression reduced by $23 \%(P<0.05)$ in the LT group. In the EDL, expression increased by $38 \%(P<0.05)$ in the ET group and decreased by $35 \%(P<0.05)$ in the LT group compared with the SA group. In the GAS, expression increased significantly by $50 \%$ in the PRE group and $29 \%$ in the ET group $(P<0.05)$ compared with the SA group (Figure 5 c).

The relative sIn mRNA expression levels were comparable in the three muscles, with levels similar in the POST and SA groups, but significantly lower in the other groups. In the SOL, no significant differences were found among the four groups, but expression levels were $36 \%-42 \%$ ( $P<$ 0.05) lower than that in the SA group. In the EDL, expression decreased significantly by $54 \%$ in the PRE, $81 \%$ in the LT, $66 \%$ in the IBA, and $74 \%$ in the ET (all $P<0.001$ ) compared with the SA group. In the GAS, no significant differences in expression levels were observed among the three hibernation groups, with levels $43 \%-47 \%(P<0.05)$ lower than that in the SA group (Figure $5 d)$.

Relative expression levels of cam and csq1 are shown in Figures 5 e and f, respectively. In the SOL, the relative csq1 mRNA expression was lower in the five groups compared with the SA group (16\% in the PRE, 14\% in the LT, $27 \%$ in the IBA, $22 \%$ in the ET $(P<0.05)$, and $64 \%$ in the POST $(P<$ $0.001)$. In the EDL, only expression levels in the PRE (39\%, $P<0.01)$, IBA $(26 \%, P<0.05)$, and POST groups $(57 \%, P<0.001)$ were lower than that in the SA group, with no significant differences found among the other groups. In the GAS, except for the PRE group, expression was higher in all hibernating groups than in the SA group, with increases of $26 \%(P<0.05)$ in the LT, $40 \%(P<0.05)$ in the IBA, 63\% $(P<0.05)$ in the ET, and $47 \%(P$ $<0.05)$ in the POST (Figure 5e).

In the SOL, the relative cam 1 mRNA expression increased by $41 \%(P<0.05)$ in the PRE group and decreased by $75 \%(P<0.05)$ in the POST group compared with the SA group. In the EDL, expression was significantly higher in the PRE $(95 \%, P<0.05), \mathrm{IBA}(204 \%, P<0.01), \mathrm{ET}(255 \%, P<$ 0.001), and POST groups $(55 \%, P<0.05)$ compared with the SA group. In the GAS, expression was $36 \%-75 \%(P<0.05)$ lower in the ET, LT, and POST groups compared with the SA groups (Figure $5 f$ ).

Relative expression levels of ryr1 and $f k b p 12$ are shown in Figures $5 \mathrm{~g}$ and h, respectively. In the SOL, the relative ryr $1 \mathrm{mRNA}$ expression increased by $21 \%(P<0.05)$ in the IBA group but decreased by $29 \%(P<0.05)$ in the ET group and by $33 \%$ in the LT group $(P<0.05)$ compared with the SA group. In the EDL, expression decreased significantly by $52 \%$ in the ET group and by $54 \%$ in the LT group $(P<0.05)$ compared with the SA group. In the GAS, expression was reduced by $50 \%$ in the PRE, $48 \%$ in the ET, and $45 \%$ in the LT $(P<0.05)$ compared with the SA group (Figure $5 \mathrm{~g}$ ).

In the SOL, the relative fkbp 12 mRNA expression levels were reduced by $38 \%(P<0.05), 61 \%(P<0.01), 69 \%(P<0.01), 68 \%(P<0.01)$, and $37 \%$ $(P<0.05)$ in the PRE, LT, IBA, ET, and POST groups, respectively, compared with the SA group. In the EDL, expression was reduced by $41 \%, 43 \%$, and 25\% $(P<0.05)$ in the ET, LT, and POST groups, respectively, compared with the SA group. In the GAS, expression was significantly decreased by 64\% $(P<0.05), 62 \%(P<0.05)$, and 69\% $(P<0.05)$ in the PRE, ET, and LT groups compared with the SA group (Figure 5h).

Co-localization of regulatory proteins involved in RyR1. The reticulate subcellular distributions of DHPR and RyR1 fluorescently labeled proteins are shown in Figure 6a. The co-localization levels of DHPR and RyR1 in the LT and ET groups were significantly lower than that in the SA group in all three muscles. In the SOL, levels were decreased in all five groups compared to the SA group, with decrements of $24 \%, 32 \%, 28 \%, 38 \%$, and $19 \%(P<0.05)$ in the PRE, LT, IBA, ET, and POST groups, respectively. In the EDL, levels decreased by $25 \%, 20 \%$, and $27 \%(P<0.05)$ in the LT, IBA, and ET groups, respectively. In the GAS, levels in the LT group $(25 \%, P<0.05)$ and ET group $(31 \%, P<0.05)$ were lower than that in the SA group, whereas levels in the POST group increased $(22 \%, P<0.05)$ compared to the SA group (Figure $6 \mathrm{c})$.

The reticulate subcellular distributions of CSQ1 and RyR1 fluorescently labeled proteins are shown in Figure 6d. The co-localization levels of CSQ1 and RyR1 in the LT, IBA, and ET groups were significantly lower than that in the PRE group in all three muscles. In the SOL, the decrements were $18 \%, 15 \%$, and $11 \%(P<0.05)$ in ET, IBA, and LT groups, respectively. In the EDL, the decrements were $12 \%$ and $12 \%(P<0.05)$ in the ET and LT groups, respectively. In the GAS, the decrements were $23 \%, 18 \%$, and $24 \%(P<0.05)$ in the ET, IBA and LT groups, respectively (Figure $6 f)$. 
The reticulate subcellular distributions of FKBP12 and RyR1 fluorescently labeled proteins are shown in Figure 6g. In the SOL and EDL, the colocalization levels of FKBP12 and RyR1 showed no significant differences among the six groups. In the GAS, however, the co-localization level decreased by $11 \%(P<0.05)$ in the LT group compared with the SA group (Figure 6i).

Co-localization of regulatory proteins involved in SERCA. The reticulate subcellular distributions of SERCA1 and SLN fluorescently labeled proteins are shown in Figure 7a. Similar change trends in the co-localization levels of SERCA1 and SLN were observed in the three different muscles, with lower levels in all groups compared with that in the SA group. In the SOL, levels decreased by $21 \%, 26 \%, 33 \%$, and $31 \%$ in the PRE, LT, IBA, and ET groups, respectively $(P<0.05)$. In the EDL, levels decreased by $15 \%, 24 \%$, and $20 \%$ in the PRE, LT, and IBA groups, respectively $(P<$ 0.05). In the GAS, levels decreased by $13 \%$ and $24 \%(P<0.05)$ in PRE and ET groups, respectively, whereas all other groups were at the summer level (Figure 7c).

The reticulate subcellular distributions of SERCA2 and SLN fluorescently labeled proteins are shown in Figure 7d. Similar change trends in the colocalization levels of SERCA2 and SLN were observed in the EDL and GAS, with lower levels in all groups compared with that in the SA group. Compared to the SA group, levels in the EDL decreased by $22 \%$ and $12 \%(P<0.05)$ in LT and IBA groups, respectively; whereas, levels in the GAS decreased by $31 \%$ and $24 \%(P<0.05)$ in PRE and ET groups, respectively. In the SOL, levels increased by $12 \%$ and $14 \%(P<0.05)$ in LT and POST groups, respectively, compared to the SA group (Figure $7 f$ ).

\section{Discussion}

We studied the cytosolic and SR $\mathrm{Ca}^{2+}$ concentrations in skeletal muscle fibers during different hibernation periods, as well as the regulation mechanism of $\mathrm{SR} \mathrm{Ca}^{2+}$ in the maintenance of cytoplasmic $\mathrm{Ca}^{2+}$ homeostasis. Results showed that, in the late stage of hibernation, there was obvious cytosolic $\mathrm{Ca}^{2+}$ overload in the three skeletal muscle fibers, but levels completely or partially recovered to summer levels after inter-bout arousal. Thus, hibernating ground squirrels exhibited the ability to alleviate cytosolic $\mathrm{Ca}^{2+}$ overload in their skeletal muscles. The protein expression levels of RyR1, SERCA1, and two $\mathrm{Ca}^{2+}{ }^{+}$-binding proteins (CSQ1 in the SR and CaM in the cytoplasm) increased significantly in the three skeletal muscles, indicating that the $\mathrm{Ca}^{2+}$ regulatory potential of the SR increased markedly during hibernation. More importantly, the opening probability of RyR1 were determined by the co-localization of DHPR, CSQ1, and FKBP12 with RyR1, and explored the regulatory mechanism of $\mathrm{Ca}^{2+}$ pump activity in hibernation via the co-localization of SLN and SERCA1/2, PLB phosphorylation level, and PLB upstream signaling pathway (Figure 8).

The $\mathrm{Ca}^{2+}$ concentration showed a $106 \%-213 \%(P<0.001)$ overload in the cytoplasm, but a significant reduction $(68 \%-80 \%, P<0.001)$ in the SR in the three different skeletal muscles in late torpor compared to levels in the summer group; however, both concentrations recovered completely or partially in early torpor. This indicates that skeletal muscle fibers in hibernating ground squirrels possess a remarkable ability to maintain Ca ${ }^{2+}$ homeostasis in the cytoplasm. The cytoplasmic and SR Ca ${ }^{2+}$ concentrations fluctuated periodically during hibernation and showed the opposite changes. This suggested that the flow of $\mathrm{Ca}^{2+}$ ions between the cytoplasm and SR in skeletal muscle fibers was very active during different periods of hibernation. Here, during late torpor, resting free $\mathrm{Ca}^{2+}$ concentration overload occurred in all three skeletal muscle fibers, similar to the cytoplasmic $\mathrm{Ca}^{2+}$ concentration overload detected in the SOL of hibernating European hamsters (Cricetus cricetus) [66]. While previous research reported a $\mathrm{Ca}^{2+}$ overload of $117 \%$ in the SOL muscle of non-hibernating mice after $7 \mathrm{~d}$ of hindlimb immobilization [9].This suggests that hibernators may also experience similar cytoplasmic $\mathrm{Ca}^{2+}$ overload as found in the disuse state in non-hibernators. However, unlike nonhibernating animals, the $\mathrm{Ca}^{2+}$ concentrations in the cytoplasm and SR of the three distinct skeletal muscles after interbout arousal (early torpor group) were restored to summer levels in the short $12-36 \mathrm{~h}$ of inter-bout arousal. This strongly suggests that the torpor-arousal cycle plays an important role in the recovery of intracellular $\mathrm{Ca}^{2+}$ homeostasis in skeletal muscle fibers of hibernating ground squirrels.

Importantly, we also found that RyR1 protein expression was significantly increased $(37 \%-107 \%, P<0.05)$ in the three muscles during hibernation (late torpor, inter-bout arousal, and early torpor groups) compared to levels in the summer and pre-hibernation groups. This indicated that the potential ability of the SR to release $\mathrm{Ca}^{2+}$ increased and may be one reason for the cytosolic $\mathrm{Ca}^{2+}$ overload and $\mathrm{SR} \mathrm{Ca}^{2+}$ decrease during late torpor and inter-bout arousal. RyR1 is the most important $\mathrm{Ca}^{2+}$-releasing pathway in the SR. Earlier studies have shown that RyR1 protein expression increases (1-2 times) in the SOL and GAS muscles of hindlimb-unloading rats, which may be one of the mechanisms of muscle atrophy caused by $\mathrm{Ca}^{2+}$ overload $[24,41]$. Thus, it may be that the increase in RyR1 protein expression in the SR is a common mechanism of $\mathrm{Ca}^{2+}$ overload in the skeletal muscle fibers in hibernating and non-hibernating animals.

In this study, the co-localization levels of DHPR and RyR1 in three skeletal muscles were significantly decreased in late torpor and early torpor groups compared to the summer group. As the structural combination of these two proteins is the basis for excitation-contraction coupling during skeletal muscle depolarization [34,36], this result prompts that the contraction function of skeletal muscle was weakened at this time, this is consistent with the fact that the skeletal muscles are basically inactive during hibernation. In the inter-bout arousal group, the protein colocalization level of DHPR and RyR1 showed a slight increase, though still lower than the summer level, which suggests that the contraction 
function of skeletal muscle can be slightly enhanced in this period, which was consistent with the active state of skeletal muscle during interbout arousal. In addition, in view of the significant increase in RyR1 protein expression in skeletal muscle fibers during hibernation, the significant decrease in DHPR protein expression in each group may be one of the main reasons for the decrease in the co-localization level of DHPR and RyR1 during the hibernation period. The increased free $\mathrm{Ca}^{2+}$ in SR can directly act on RyR1 to promote channel opening, whereas CSQ1 can inhibit channel opening by binding with RyR1 [37,38]. The current study showed that the SR free $\mathrm{Ca}^{2+}$ level in the three skeletal muscles during

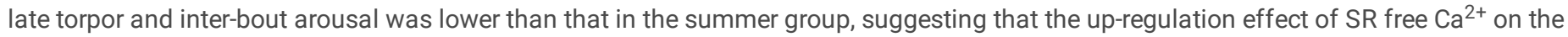
opening of RyR1 was weakened during hibernation. The protein co-localization level of CSQ1 and RyR1 in the GAS of the hibernation group was significantly lower than that in summer group, suggesting that the protein binding level of CSQ1 to RyR1 was significantly lower, and that the inhibition of CSQ1 on RyR1 was weakened during hibernation. This suggests that the decreased inhibition of CSQ1 on RyR1 over the hibernation period may increase the probability of the RyR1 channel opening, and thus may be one of the mechanisms of cytoplasmic Ca ${ }^{2+}$ overload in skeletal muscle fibers during hibernation. FKBP12 is another important regulatory factor of RyR1 in skeletal muscle. When it is combined with RyR1, the channel tends to close, and the probability of the channel opening increases when it dissociates [40,67, 68]. We found that FKBP12 protein expression increased in the SOL and EDL during hibernation groups, whereas the co-localization level between FKBP12 and RyR1 demonstrated no significant differences in the SOL and EDL during hibernation, suggesting that the inhibition of FKBP12 on RyR1 was stable during this period. Nevertheless, the decreased co-localization level between RyR1 and FKBP12 in the GAS indicates that the openness degree of RyR1 channel increased during late torpor. These results indicate that the increase in RyR1 protein expression may be the common mechanism of $\mathrm{Ca}^{2+}$ overload in the cytoplasm of three skeletal muscle fibers during the later torpor and interbout arousal period and the decrease in the inhibition of CSQ1 and FKBP12 on the RyR1 channel in GAS jointly promoted the increase in $\mathrm{Ca}^{2+}$ release in the SR during hibernation, thus constituting a multiple mechanism of cytoplasmic $\mathrm{Ca}^{2+}$ overload in GAS muscle fibers during late torpor and inter-bout arousal.

Interestingly, we found that SERCA1 protein expression also increased significantly $(28 \%-102 \%, P<0.05)$ in the three skeletal muscles during

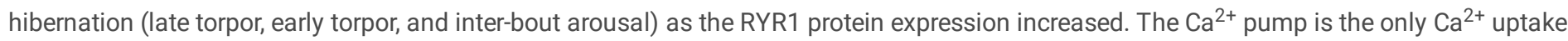
channel in the SR and expresses subtypes 1 and 2 in different skeletal muscles $[46,47]$. The increased protein expression of SERCA1 in the three muscles during hibernation suggests that the potential ability of the SR to absorb cytosolic $\mathrm{Ca}^{2+}$ was enhanced. This is consistent with our previous study, which showed increased SERCA2 protein expression in the SOL and EDL during hibernation [60], but is inconsistent with earlier research on Siberian ground squirrels (Spermophilus undulatus), which showed decreased RyR1 and SERCA1 protein expression in the SOL and EDL muscles during hibernation [27]. In the present study, the increased expression of RyR1 and SERCA1 in hibernating skeletal muscle indicated that the ability of the SR to release and uptake $\mathrm{Ca}^{2+}$ was enhanced. This further indicated that the concentration of $\mathrm{Ca}^{2+}$ in the cytoplasm and SR of skeletal muscle fibers of ground squirrels reached a dynamic balance at a relatively higher activity level during hibernation. Conversely, in hibernating Siberian ground squirrels, the concentration of $\mathrm{Ca}^{2+}$ in the cytoplasm and SR of skeletal muscle achieved a dynamic balance at a lower level [27]. This may be due to the different severity of the animals' living environments, reflecting different molecular strategies of hibernating animals to cope with long-term skeletal muscle inactivity during hibernation.

PLB is a key negative regulatory protein of the $\mathrm{Ca}^{2+}$ pump, the phosphorylation state of PLB represents the elimination of $\mathrm{Ca}^{2+}$ pump inhibition, i.e., up-regulation of $\mathrm{Ca}^{2+}$ pump activity [69-71]. Here, the protein expression of PLB increased $(19 \%-112 \%, P<0.05)$ in all three muscles over the

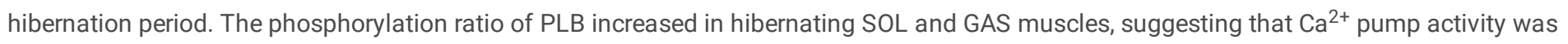
enhanced in the SOL and GAS muscles, and is in contrast to our previous research, which showed that $\mathrm{Ca}^{2+}$ pump activity increased after interbout arousal in the EDL [60]. Unlike that in the SOL and GAS, the phosphorylation level of PLB in the EDL decreased during hibernation. This difference could be attributed to the different types of muscle fibers. Previous studies have shown that SERCA1 and SLN are mainly expressed in fast skeletal muscle, with SERCA2 and PLB less expressed [45, 72]. Therefore, the effect of PLB on Ca ${ }^{2+}$ pump activity might be less than that of SLN in typical fast-twitch muscles, such as the EDL. Generally, the increased phosphorylation of PLB in the SOL and GAS during early torpor or inter-bout arousal indicates that the PLB pathway mediates the increase in $\mathrm{Ca}^{2+}$ pump activity during hibernation, which may be one of the important mechanisms against $\mathrm{Ca}^{2+}$ overload in skeletal muscle fibers during early torpor.

SLN is another key negative regulatory protein of the $\mathrm{Ca}^{2+}$ pump, but the specific regulatory mechanism of $\mathrm{SLN}^{2}$ in the Ca ${ }^{2+}$ pump is not completely clear, although the combination of $\mathrm{Ca}^{2+}$ pump with SLN is reported to inhibit $\mathrm{Ca}^{2+}$ pump activity [73-76]. Previous research has also shown that SERCA1 and SLN are mainly expressed in mammalian fast muscles, but not SERCA2 and PLB [45, 72]. Our results showed that the protein expression of SLN increased to different degrees in the three muscles during hibernation and the co-localization level between SLN and SERCA1 in the EDL decreased significantly during late torpor and inter-bout arousal, suggesting that the inhibition of SLN on the Ca ${ }^{2+}$ pump was weakened during hibernation, i.e., $\mathrm{Ca}^{2+}$ pump activity increased. This is similar to our previous study, which showed that $\mathrm{Ca}^{2+}$ pump activity in the EDL increases significantly in inter-bout arousal [60]. In the GAS, the co-localization level between SLN and SERCA (SERCA1 and SERCA2) decreased or remained unchanged in the hibernation groups, suggesting that $\mathrm{Ca}^{2+}$ pump activity was maintained or up-regulated. Generally, the inhibition of SLN on the $\mathrm{Ca}^{2+}$ pump was weakened, which may be another mechanisms against $\mathrm{Ca}^{2+}$ overload in skeletal muscle fibers of EDL and GAS during hibernation. 
We also found that $\beta$-AR2 protein expression increased significantly during the whole hibernation period in the GAS muscle, suggesting that Ca ${ }^{2+}$ pump activity was up-regulated. $\beta$-AR2 is an important G-protein-coupled receptor on the cell membrane. It can act on PLB through intracellular CAMP-PKA cascade reactions and dissociates from the $\mathrm{Ca}^{2+}$ pump after phosphorylation, thereby increasing Ca ${ }^{2+}$ pump activity [77]. Therefore, the increase in $\beta$-AR2 protein expression in all hibernation groups in the GAS may explain the increase in phosphorylation level of PLB. In the SOL and EDL, $\beta$-AR2 protein expression during hibernation was not higher than that in the summer group, suggesting that this pathway is not responsible for the up-regulation of $\mathrm{Ca}^{2+}$ pump activity in the two muscles.

In addition, CaM and increased free $\mathrm{Ca}^{2+}$ activate CaMK2, which can increases $\mathrm{Ca}^{2+}$ pump activity [78, 79]. Results showed that the phosphorylation level of P-CaMK2 increased in the SOL muscle but decreased in the GAS and EDL muscles during inter-bout arousal. This result suggests that the increased phosphorylation ratio of CaMK2 may be an upstream signaling factor for the increase in slow-twitch SOL muscle $\mathrm{Ca}^{2+}$ pump activity during hibernation. This differs from the increase in $\mathrm{Ca}^{2+}$ pump activity observed in the GAS during hibernation, which appeared to be mainly regulated by $\beta$-AR2. This suggests that the up-regulation mechanism of calcium pump activity of skeletal muscle fibers during hibernation exhibit muscle fiber type specificity among different muscles.

We also found that that the expression of two $\mathrm{Ca}^{2+}$-binding proteins, i.e., CaM in the cytoplasm and CSQ1 in the SR, increased in almost all hibernating periods (late torpor, inter-bout arousal, and early torpor). Each CaM molecule can bind with four $\mathrm{Ca}^{2+}$ in the cytoplasm [56, 57]; thus, in the present study, the increase in protein expression observed during hibernation undoubtedly helped to reduce the concentration of free $\mathrm{Ca}^{2+}$ in the cytoplasm. This is similar to results reported in the SOL and GAS muscles of thirteen-lined ground squirrels, which showed increased protein expression during hibernation [28, 80]. CSQ molecules can bind with $43 \mathrm{Ca}^{2+}$ in the SR $[38,55,58,59]$; thus, in our study, the increased CSQ1 protein expression observed during hibernation indicated that the ability of skeletal muscle fibers to reduce the free Ca ${ }^{2+}$ concentration in the SR was increased. Previous studies have shown that the resultant reduction in free $\mathrm{Ca}^{2+}$ in the SR contributes to the reduction in Ca ${ }^{2+}$ release [18]. Therefore, the high expression of CSQ1 in the SR and CaM in the cytoplasm during hibernation undoubtedly inhibited the increase in cytosolic free $\mathrm{Ca}^{2+}$ and participated in the maintenance of cytosolic $\mathrm{Ca}^{2+}$ homeostasis. In addition, the cytoplasmic and $\mathrm{SR} \mathrm{Ca}^{2+}$ concentrations in pre-hibernation were lowest among almost all groups. This suggests that the increased CaM and CSQ1 protein expression levels could combine with the free $\mathrm{Ca}^{2+}$ in the cytoplasm and SR, respectively, during hibernation. This increase in protein expression provides a doubleinsurance mechanism to reduce the free $\mathrm{Ca}^{2+}$ concentration in the cytoplasm and $\mathrm{SR}$, and therefore avoid cytoplasmic $\mathrm{Ca}^{2+}$ overload in skeletal muscle of ground squirrels during hibernation.

\section{Conclusion}

In summary, we carried out a comprehensive study to explore the mechanisms of SR $\mathrm{Ca}^{2+}$ regulation in the maintenance of cytosolic Ca ${ }^{2+}$ homeostasis in skeletal muscle fibers of hibernating Daurian ground squirrels. Calcium overload in skeletal muscle occurred in hibernating ground squirrels during late torpor, similar to that in non-hibernators in the disuse state; however, its recovery in early torpor indicated that hibernating ground squirrels have a remarkable ability to alleviate cytosolic $\mathrm{Ca}^{2+}$ overload in skeletal muscle cytoplasm. The significant increase in RyR1 protein expression and the decreased inhibition of CSQ1 and FKBP12 on RyR1 in skeletal muscles during hibernation also indicated that the SR has an enhanced ability to release $\mathrm{Ca}^{2+}$, which may be an important mechanism for $\mathrm{Ca}^{2+}$ overload during inter-bout arousal. The colocalization level of DHPR and RyR1 decreased during hibernation, indicating a decrease in excitation contraction coupling, consistent with the decrease in contraction activity of skeletal muscles during hibernation. However, the protein expression of SERCA1 increased significantly during hibernation and the inhibition of PLB and SLN on SERCA decreased significantly. That is, the protein expression level of SERCA1 increased synchronously with SERCA1 activity during hibernation, suggesting that the ability of skeletal muscle SR to absorb Ca ${ }^{2+}$ increased significantly during hibernation, which may be an important mechanism to alleviate cytosolic $\mathrm{Ca}^{2+}$ overload. Moreover, the increase in CaMK2 phosphorylation level during hibernation may be one of the mechanism to increase activity of SERCA in the slow SOL muscle, whereas the decrease in inhibition of PLB on SERCA in the GAS may be due to the increase in $\beta$-AR2 protein expression. The expression of CSQ1 in the SR and $\mathrm{CaM}$ in the cytoplasm increased significantly, indicating that the ability of muscle fibers to reduce intracellular free $\mathrm{Ca}^{2+}$ concentration was enhanced during hibernation. These results suggest that the enhanced release of $\mathrm{Ca}^{2+}$ from the SR may be the main mechanism leading to $\mathrm{Ca}^{2+}$ overload in hibernation. Thus, the enhanced $\mathrm{Ca}^{2+}$ pump protein expression and activity are important mechanisms for reducing $\mathrm{Ca}^{2+}$ overload and restoring intracellular $\mathrm{Ca}^{2+}$ homeostasis. These findings confirm, for the first time, that the SR in skeletal muscle cells may be more active during hibernation (hypothermic state) than during non-hibernation (homoiothermic state). Up-regulation of $\mathrm{Ca}^{2+}$ pump activity in the SR via different signaling pathways, plus the dramatic increase in CaM and CSQ1 expression combined with free $\mathrm{Ca}^{2+}$, constitute the major mechanisms of hibernators to maintain intracellular $\mathrm{Ca}^{2+}$ homeostasis during hibernation.

\section{Abbreviations}


Sarcoplasmic reticulum

$\mathrm{Ca}^{2+}$

Calcium

SOL

Soleus

EDL

Extensor digitorum longus

GAS

Gastrocnemius

RyR1

Ryanodine receptor1

SERCA1

Sarco/endoplasmic reticulum Ca2 + ATPase isoform 1

DHPR

Dihydropyridine receptor

CSQ1

Calsequestrin1

FKBP12

12-kDa FK506 binding protein

PLB

Phospholamban

SLN

Sarcolipin

$\beta$-AR2

$\beta$-adrenergic receptor2

CaMK2

Calmodulin kinase2

CaM

Calmodulin

SA

Summer active

PRE

Pre-hibernation

LT

Late torpor

IBA

Interbout arousal

ET

Early torpor

POST

Post-hibernation

Fluo-3/AM

Fluo-3-acetoxymethylester

mag-Fluo-4/AM

Magnesium-Fluo-4-acetoxymethylester

DAPI

4'-6'-diamidino-2-phenylindole

RT-PCR

Real-time PCR

MWW

Skeletal muscle wet weight

MWW/BW

Ratio of skeletal muscle wet weight to body weight

\section{Declarations}


All animal procedures and care and handling protocols were approved by the Committee on the Ethics of Animal Experiments of the Northwest University (Permit Number: SYXK 2010-004).

\section{Consent for publication}

Not applicable.

\section{Availability of data and material}

The datasets supporting the conclusions of this article are included within the article and its additional file.

\section{Funding}

This work was supported by funds from the National Natural Science Foundation of China (No. 31772459) and the Shaanxi Province Natural Science Basic Research Program (2018JM3015).

\section{Competing interests}

The authors declare that they have no competing interests.

\section{Authors' contributions}

Conceived and designed the experiments: YFG, ZW, HPW,

Performed the experiments: ZW, XFM, JZ, HC, PX, SHX,

Analyzed the data: ZW, XFM,

Wrote the manuscript: ZW, JZ, YFG.

\section{Acknowledgements}

Not applicable

\section{Tables}

Table 1. Primary and secondary antibodies used in protein co-localization.

\begin{tabular}{|c|c|c|}
\hline Protein type & Primary antibody cocktails and concentrations & Secondary antibody cocktails and concentrations \\
\hline \multirow[t]{2}{*}{ DHPR/RyR1 } & rabbit anti-RyR1 (1:200, \#8153, CST) & $\begin{array}{l}\text { goat anti-rabbit Alexa Fluor 647(1:200, \#A21245, Thermo Fisher } \\
\text { Scientific) }\end{array}$ \\
\hline & $\begin{array}{l}\text { mouse anti-DHPR (1:200, \#SC21781, Santa Cruz, CA, } \\
\text { USA) }\end{array}$ & goat anti-mouse FITC (1:200, \# F1010, Sigma-Aldrich) \\
\hline \multirow[t]{2}{*}{ CSQ1/RyR1 } & rabbit anti-RyR1 & goat anti-rabbit Alexa Fluor 647 \\
\hline & $\begin{array}{l}\text { rabbit anti-calsequestrin } 1 \text { (1:100, \#ab191564, } \\
\text { Abcam) }\end{array}$ & $\begin{array}{l}\text { goat anti-rabbit Alexa Fluor 488(1:200, \#11034, Thermo Fisher } \\
\text { Scientific) }\end{array}$ \\
\hline \multirow[t]{2}{*}{ FKBP12/RyR1 } & rabbit anti-RyR1 & goat anti-rabbit Alexa Fluor 647 \\
\hline & mouse anti-FKBP12 (1:200, ab58072, Abcam) & goat anti-mouse FITC \\
\hline \multirow[t]{2}{*}{ SLN/SERCA1 } & rabbit anti-SERCA1 (1:200, \#4219, CST) & goat anti-rabbit Alexa Fluor 647 \\
\hline & rabbit anti-SLN (1:200, \# 18395-1-AP, Proteintech) & goat anti-rabbit Alexa Fluor 488 \\
\hline \multirow[t]{2}{*}{ SLN/SERCA2 } & rabbit anti-SERCA2 (1:200, \#4388, CST) & goat anti-rabbit Alexa Fluor 647 \\
\hline & rabbit anti-SLN & goat anti-rabbit Alexa Fluor 488 \\
\hline
\end{tabular}

Table 2. Effects of hibernation on body weight (BW), muscle wet weight (MWW), and ratio of MWW/BW in Daurian ground squirrels. 


\begin{tabular}{|c|c|c|c|c|c|c|c|c|}
\hline \multirow[t]{2}{*}{ Group } & \multirow{2}{*}{$\begin{array}{l}\text { BW before } \\
\text { hibernation } \\
\text { (g) }\end{array}$} & \multirow{2}{*}{$\begin{array}{l}\text { BW at experiment } \\
\text { (g) }\end{array}$} & \multicolumn{3}{|c|}{ MWW at experiment (mg) } & \multicolumn{3}{|c|}{ MWW/BW at experiment $(\mathrm{mg} / \mathrm{g})$} \\
\hline & & & SOL & EDL & GAS & SOL & EDL & GAS \\
\hline SA & $325.24 \pm 27.46$ & $325.24 \pm 27.46$ & $131 \pm 18$ & $140 \pm 18$ & $257 \pm 31$ & $0.40 \pm 0.05$ & $0.43 \pm 0.04$ & $0.79 \pm 0.10$ \\
\hline PRE & $315.44 \pm 21.82$ & $315.44 \pm 21.82$ & $101 \pm 23^{*}$ & $123 \pm 27$ & $261 \pm 41$ & $0.32 \pm 0.03^{\star \star \star}$ & $0.39 \pm 0.05$ & $0.82 \pm 0.09$ \\
\hline LT & $322.71 \pm 18.49$ & 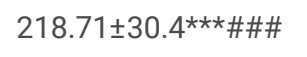 & $99 \pm 19 *$ & $110 \pm 32^{\star}$ & $211 \pm 27 * \#$ & $0.45 \pm 0.05^{\star} \# \#$ & $0.50 \pm 0.06 \#$ & $0.96 \pm 0.12^{\star \star \# \# ~}$ \\
\hline IBA & $330.14 \pm 26.59$ & $232.71 \pm 32.07 \star \star \star \# \# \#$ & $113 \pm 24^{*}$ & $118 \pm 22^{*}$ & $225 \pm 28^{\star} \#$ & $0.49 \pm 0.04^{\star} \# \#$ & $0.51 \pm 0.07 \#$ & $0.97 \pm 0.11^{\star *} \# \#$ \\
\hline ET & $319.51 \pm 24.90$ & $217.83 \pm 25.70^{\star \star \star \# \# \# ~}$ & $102 \pm 18^{*}$ & $114 \pm 19 *$ & $195 \pm 32^{\star \star \# \# ~}$ & $0.47 \pm 0.03^{\star \# \#}$ & $0.53 \pm 0.04^{\star} \# \#$ & $0.90 \pm 0.08^{\star} \#$ \\
\hline POST & $319.39 \pm 30.15$ & $223.20 \pm 31.43^{\star \star \star \# \# \# ~}$ & $101 \pm 15^{\star}$ & $112 \pm 23^{*}$ & $200 \pm 34^{\star} \#$ & $0.45 \pm 0.06^{*} \# \#$ & $0.50 \pm 0.04 \#$ & $0.90 \pm 0.08^{\star} \#$ \\
\hline
\end{tabular}

SOL, soleus; GAS, gastrocnemius; EDL, extensor digitorum longus; SA, summer active; PRE, pre-hibernation; LT, late torpor; IBA, inter-bout arousal; ET, early torpor; POST, post-hibernation. Data represent means $\pm \mathrm{SD} ; \mathrm{n}=8 .{ }^{\star} P<0.05$ compared with $\mathrm{SA} ;{ }^{* \star} P<0.01$ compared with $\mathrm{SA}$; ${ }^{* \star} P<$ 0.001 compared with SA. ${ }^{\#} P<0.05$ compared with PRE; ${ }^{*} P<0.01$ compared with PRE; ${ }^{\# \#} P<0.001$ compared with PRE.

\section{References}

1. Ghosh A, Greenberg ME: Calcium signaling in neurons: molecular mechanisms and cellular consequences. Science 1995, 268(5208):239247.

2. Hardingham GE, Bading H: Nuclear calcium: a key regulator of gene expression. Biometals : an international journal on the role of metal ions in biology, biochemistry, and medicine 1998, 11(4):345-358.

3. Thomas AP, Bird GS, Hajnoczky G, Robb-Gaspers LD, Putney JW, Jr.: Spatial and temporal aspects of cellular calcium signaling. Faseb J 1996, 10(13):1505-1517.

4. Costelli P, Reffo P, Penna F, Autelli R, Bonelli G, Baccino FA: Ca2+-dependent proteolysis in muscle wasting. Int J Biochem Cell B 2005, 37(10):2134-2146.

5. Goll DE, Thompson VF, Li H, Wei W, Cong J: The calpain system. Physiological reviews 2003, 83(3):731-801.

6. Shenkman BS, Nemirovskaya TL: Calcium-dependent signaling mechanisms and soleus fiber remodeling under gravitational unloading. Journal of muscle research and cell motility 2008, 29(6-8):221-230.

7. Fu WW, Hu HX, Dang K, Chang H, Du B, Wu X, Gao YF: Remarkable preservation of Ca2+ homeostasis and inhibition of apoptosis contribute to anti-muscle atrophy effect in hibernating Daurian ground squirrels. Sci Rep-Uk 2016, 6:13.

8. Goll DE, Neti G, Mares SW, Thompson VF: Myofibrillar protein turnover: the proteasome and the calpains. Journal of animal science 2008, 86(14 Suppl):E19-35.

9. Ingalls CP, Wenke JC, Armstrong RB: Time course changes in [Ca2+]i, force, and protein content in hindlimb-suspended mouse soleus muscles. Aviation, space, and environmental medicine 2001, 72(5):471-476.

10. Hu NF, Chang H, Du B, Zhang QW, Arfat Y, Dang K, Gao YF: Tetramethylpyrazine ameliorated disuse-induced gastrocnemius muscle atrophy in hindlimb unloading rats through suppression of $\mathrm{Ca}(2+) / \mathrm{ROS}$-mediated apoptosis. Applied physiology, nutrition, and metabolism = Physiologie appliquee, nutrition et metabolisme 2017, 42(2):117-127.

11. Wu X, Gao YF, Zhao XH, Cui JH: [Effects of tetramethylpyrazine on nitric oxide synthase activity and calcium ion concentration of skeletal muscle in hindlimb unloading rats]. Zhonghua yi xue za zhi 2012, 92(29):2075-2077.

12. Gao YF, Arfat Y, Wang HP, Goswami N: Muscle Atrophy Induced by Mechanical Unloading: Mechanisms and Potential Countermeasures. Front Physiol 2018, 9:17.

13. Carey HV, Andrews MT, Martin SL: Mammalian hibernation: cellular and molecular responses to depressed metabolism and low temperature. Physiological reviews 2003, 83(4):1153-1181.

14. Gao YF, Wang J, Wang HP, Feng B, Dang K, Wang Q, Hinghofer-Szalkay HG: Skeletal muscle is protected from disuse in hibernating dauria ground squirrels. Comp Biochem Phys A 2012, 161(3):296-300.

15. Rourke BC, Yokoyama Y, Milsom WK, Caiozzo VJ: Myosin isoform expression and MAFbx mRNA levels in hibernating golden-mantled ground squirrels (Spermophilus lateralis). Physiological and biochemical zoology : PBZ 2004, 77(4):582-593.

16. Lebiedzinska M, Szabadkai G, Jones AW, Duszynski J, Wieckowski MR: Interactions between the endoplasmic reticulum, mitochondria, plasma membrane and other subcellular organelles. The international journal of biochemistry \& cell biology 2009, 41(10):1805-1816.

17. Pinto MC, Kihara AH, Goulart VA, Tonelli FM, Gomes KN, Ulrich H, Resende RR: Calcium signaling and cell proliferation. Cellular signalling 2015, 27(11):2139-2149. 
18. Allen DG, Kabbara AA, Westerblad H: Muscle fatigue: the role of intracellular calcium stores. Canadian journal of applied physiology = Revue canadienne de physiologie appliquee 2002, 27(1):83-96.

19. Maclntosh BR, Holash RJ, Renaud JM: Skeletal muscle fatigue - regulation of excitation-contraction coupling to avoid metabolic catastrophe. J Cell Sci 2012, 125(9):2105-2114.

20. Pozzan T, Rizzuto R, Volpe P, Meldolesi J: Molecular and cellular physiology of intracellular calcium stores. Physiological reviews 1994, 74(3):595-636.

21. Tupling AR: The sarcoplasmic reticulum in muscle fatigue and disease: role of the sarco(endo)plasmic reticulum Ca2+-ATPase. Canadian journal of applied physiology = Revue canadienne de physiologie appliquee 2004, 29(3):308-329.

22. Donoghue P, Ribaric S, Moran B, Cebasek V, Erzen I, Ohlendieck K: Early effects of denervation on Ca(2+)-handling proteins in skeletal muscle. International journal of molecular medicine 2004, 13(6):767-772.

23. Hunter RB, Mitchell-Felton H, Essig DA, Kandarian SC: Expression of endoplasmic reticulum stress proteins during skeletal muscle disuse atrophy. American journal of physiology Cell physiology 2001, 281(4):C1285-1290.

24. Kraner SD, Wang Q, Novak KR, Cheng D, Cool DR, Peng J, Rich MM: Upregulation of the CaV 1.1-ryanodine receptor complex in a rat model of critical illness myopathy. American journal of physiology Regulatory, integrative and comparative physiology 2011, 300(6):R1384-1391.

25. Lamboley CR, Wyckelsma VL, Perry BD, McKenna MJ, Lamb GD: Effect of 23-day muscle disuse on sarcoplasmic reticulum Ca2+ properties and contractility in human type I and type II skeletal muscle fibers. J Appl Physiol (1985) 2016, 121(2):483-492.

26. Peters DG, Mitchell-Felton H, Kandarian SC: Unloading induces transcriptional activation of the sarco(endo)plasmic reticulum Ca2+-ATPase 1 gene in muscle. Am J Physiol 1999, 276(5 Pt 1):C1218-1225.

27. Malysheva AN, Storey KB, Ziganshin RK, Lopina OD, Rubtsov AM: Characteristics of sarcoplasmic reticulum membrane preparations isolated from skeletal muscles of active and hibernating ground squirrel Spermophilus undulatus. Biochem-Moscow 2001, 66(8):918-925.

28. Zhang YC, Storey KB: Regulation of gene expression by NFAT transcription factors in hibernating ground squirrels is dependent on the cellular environment. Cell Stress Chaperon 2016, 21(5):883-894.

29. Fabiato A: Calcium-induced release of calcium from the cardiac sarcoplasmic reticulum. Am J Physio/ 1983, 245(1):C1-14.

30. Hanson MG, Wilde JJ, Moreno RL, Minic AD, Niswander L: Potassium dependent rescue of a myopathy with core-like structures in mouse. eLife 2015, 4.

31. Laver DR: Ca2+ stores regulate ryanodine receptor Ca2+ release channels via luminal and cytosolic Ca2+ sites. Biophysical journal 2007, 92(10):3541-3555.

32. Meissner G: Regulation of mammalian ryanodine receptors. Frontiers in bioscience : a journal and virtual library 2002, 7:d2072-2080.

33. Meissner G: The structural basis of ryanodine receptor ion channel function. The Journal of general physiology 2017, 149(12):1065-1089.

34. Calderon JC, Bolanos P, Caputo C: The excitation-contraction coupling mechanism in skeletal muscle. Biophysical reviews 2014, 6(1):133160.

35. Protasi F: Structural interaction between RYRs and DHPRs in calcium release units of cardiac and skeletal muscle cells. Frontiers in bioscience : a journal and virtual library 2002, 7:d650-658.

36. Laver DR: Regulation of the RyR channel gating by Ca(2+) and Mg(2). Biophysical reviews 2018, 10(4):1087-1095.

37. Lee YS, Keener JP: A calcium-induced calcium release mechanism mediated by calsequestrin. Journal of theoretical biology 2008, 253(4):668-679.

38. Beard NA, Laver DR, Dulhunty AF: Calsequestrin and the calcium release channel of skeletal and cardiac muscle. Progress in biophysics and molecular biology 2004, 85(1):33-69.

39. Lam E, Martin MM, Timerman AP, Sabers C, Fleischer S, Lukas T, Abraham RT, O'Keefe SJ, O'Neill EA, Wiederrecht GJ: A novel FK506 binding protein can mediate the immunosuppressive effects of FK506 and is associated with the cardiac ryanodine receptor. J Biol Chem 1995, 270(44):26511-26522.

40. Timerman AP, Onoue H, Xin HB, Barg S, Copello J, Wiederrecht G, Fleischer S: Selective binding of FKBP12.6 by the cardiac ryanodine receptor. J Biol Chem 1996, 271(34):20385-20391.

41. Bastide B, Conti A, Sorrentino V, Mounier Y: Properties of ryanodine receptor in rat muscles submitted to unloaded conditions. Biochem Biophys Res Commun 2000, 270(2):442-447.

42. Bublitz M, Musgaard M, Poulsen H, Thogersen L, Olesen C, Schiott B, Morth JP, Moller JV, Nissen P: Ion pathways in the sarcoplasmic reticulum Ca2+-ATPase. J Biol Chem 2013, 288(15):10759-10765.

43. Espinoza-Fonseca LM: The $\mathrm{Ca}(2+)$-ATPase pump facilitates bidirectional proton transport across the sarco/endoplasmic reticulum. Molecular bioSystems 2017, 13(4):633-637.

44. Inesi G: Mechanism of calcium transport. Annual review of physiology 1985, 47:573-601. 
45. Periasamy M, Kalyanasundaram A: SERCA pump isoforms: Their role in calcium transport and disease. Muscle Nerve 2007, 35(4):430-442.

46. Sanchez GA, Trinks PW, Richard SB, Di Croce DE, Takara D: Expression of sarcoplasmic-endoplasmic reticulum Ca-ATPase isoforms in masticatory muscles. European journal of oral sciences 2014, 122(1):36-41.

47. Stammers AN, Susser SE, Hamm NC, Hlynsky MW, Kimber DE, Kehler DS, Duhamel TA: The regulation of sarco(endo)plasmic reticulum calcium-ATPases (SERCA). Canadian journal of physiology and pharmacology 2015, 93(10):843-854.

48. Asahi M, Sugita Y, Kurzydlowski K, De Leon S, Tada M, Toyoshima C, MacLennan DH: Sarcolipin regulates sarco(endo)plasmic reticulum Ca2+-ATPase (SERCA) by binding to transmembrane helices alone or in association with phospholamban. Proc Natl Acad Sci U S A 2003, 100(9):5040-5045.

49. Aschar-Sobbi R, Emmett TL, Kargacin GJ, Kargacin ME: Phospholamban phosphorylation increases the passive calcium leak from cardiac sarcoplasmic reticulum. Pflugers Arch 2012, 464(3):295-305.

50. Haghighi K, Gregory KN, Kranias EG: Sarcoplasmic reticulum Ca-ATPase-phospholamban interactions and dilated cardiomyopathy. Biochem Biophys Res Commun 2004, 322(4):1214-1222.

51. Nelson OL, Robbins CT, Bentjen S: Upregulation of beta 1, beta 2 and beta 3 adrenergic receptor expression in the hibernating bear myocardium: A role for cardioprotection? Faseb J 2010, 24.

52. Karczewski P, Kuschel M, Baltas LG, Bartel S, Krause EG: Site-specific phosphorylation of a phospholamban peptide by cyclic nucleotide- and Ca2+/calmodulin-dependent protein kinases of cardiac sarcoplasmic reticulum. Basic research in cardiology 1997, 92 Suppl 1:37-43.

53. Mishra S, Sabbah HN, Jain JC, Gupta RC: Reduced Ca2+-calmodulin-dependent protein kinase activity and expression in LV myocardium of dogs with heart failure. Am J Physiol Heart Circ Physiol 2003, 284(3):H876-883.

54. Li SY, Yang Z, Gao YF, Li GY, Wang HP, Hinghofer-Szalkay HG: Ligustrazine and the Contractile Properties of Soleus Muscle in HindlimbUnloaded Rats. Aviat Space Envir Md 2012, 83(11):1049-1054.

55. Prins D, Michalak M: Organellar calcium buffers. Cold Spring Harbor perspectives in biology 2011, 3(3).

56. Klee CB, TC V: Calmodulin. Annual Review of Biochemistry 1982, 35(1):213.

57. Zhang M, Tanaka T, Ikura M: Calcium-induced conformational transition revealed by the solution structure of apo calmodulin. Nature structural biology 1995, 2(9):758-767.

58. Mekahli D, Bultynck G, Parys JB, De Smedt H, Missiaen L: Endoplasmic-reticulum calcium depletion and disease. Cold Spring Harbor perspectives in biology 2011, 3(6).

59. Royer L, Rios E: Deconstructing calsequestrin. Complex buffering in the calcium store of skeletal muscle. The Journal of physiology 2009 , 587(Pt 13):3101-3111.

60. Guo Q, Mi X, Sun X, Li X, Fu W, Xu S, Wang Q, Arfat Y, Wang H, Chang H et al: Remarkable plasticity of Na(+), K(+)-ATPase, Ca(2+)-ATPase and SERCA contributes to muscle disuse atrophy resistance in hibernating Daurian ground squirrels. Sci Rep 2017, 7(1):10509.

61. Yang CX, He Y, Gao YF, Wang HP, Goswami N: Changes in calpains and calpastatin in the soleus muscle of Daurian ground squirrels during hibernation. Comparative biochemistry and physiology Part A, Molecular \& integrative physiology 2014, 176:26-31.

62. Wang Z, Jiang SF, Cao J, Liu K, Xu SH, Arfat Y, Guo QL, Chang H, Goswami N, Hinghofer-Szalkay H et al: Novel findings on ultrastructural protection of skeletal muscle fibers during hibernation of Daurian ground squirrels: Mitochondria, nuclei, cytoskeleton, glycogen. $J$ Cell Physiol 2019, 234(8):13318-13331.

63. Nakai J, Ohkura M, Imoto K: A high signal-to-noise Ca(2+) probe composed of a single green fluorescent protein. Nature biotechnology 2001, 19(2):137-141.

64. Park MK, Petersen $\mathrm{OH}$, Tepikin AV: The endoplasmic reticulum as one continuous Ca2+ pool: visualization of rapid Ca2+ movements and equilibration. Embo J 2000, 19(21):5729-5739.

65. Lagache T, Sauvonnet N, Danglot L, Olivo-Marin JC: Statistical analysis of molecule colocalization in bioimaging. Cytometry Part $A$ : the journal of the International Society for Analytical Cytology 2015, 87(6):568-579.

66. Agostini B, De Martino L, Soltau B, Hasselbach W: The modulation of the calcium transport by skeletal muscle sarcoplasmic reticulum in the hibernating European hamster. Zeitschrift fur Naturforschung C, Journal of biosciences 1991, 46(11-12):1109-1126.

67. Fill M, Copello JA: Ryanodine receptor calcium release channels. Physiological reviews 2002, 82(4):893-922.

68. Marx SO, Reiken S, Hisamatsu Y, Jayaraman T, Burkhoff D, Rosemblit N, Marks AR: PKA phosphorylation dissociates FKBP12.6 from the calcium release channel (ryanodine receptor): defective regulation in failing hearts. Cell 2000, 101(4):365-376.

69. MacLennan DH, Kranias EG: Phospholamban: a crucial regulator of cardiac contractility. Nature reviews Molecular cell biology 2003, 4(7):566-577.

70. Shaikh SA, Sahoo SK, Periasamy M: Phospholamban and sarcolipin: Are they functionally redundant or distinct regulators of the Sarco(Endo)Plasmic Reticulum Calcium ATPase? J Mol Cell Cardiol 2016, 91:81-91. 
71. Nicolaou P, Hajjar RJ, Kranias EG: Role of protein phosphatase-1 inhibitor-1 in cardiac physiology and pathophysiology. J Mol Cell Cardiol 2009, 47(3):365-371.

72. Grover AK, Khan I: Calcium pump isoforms: diversity, selectivity and plasticity. Review article. Cell calcium 1992, $13(1): 9-17$.

73. Akin BL, Chen Z, Jones LR: Superinhibitory phospholamban mutants compete with Ca2+ for binding to SERCA2a by stabilizing a unique nucleotide-dependent conformational state. J Biol Chem 2010, 285(37):28540-28552.

74. Asahi M, Kurzydlowski K, Tada M, MacLennan DH: Sarcolipin inhibits polymerization of phospholamban to induce superinhibition of sarco(endo)plasmic reticulum Ca2+-ATPases (SERCAs). J Biol Chem 2002, 277(30):26725-26728.

75. Chen Z, Stokes DL, Rice WJ, Jones LR: Spatial and dynamic interactions between phospholamban and the canine cardiac Ca2+ pump revealed with use of heterobifunctional cross-linking agents. J Biol Chem 2003, 278(48):48348-48356.

76. Lee AG: A calcium pump made visible. Current opinion in structural biology 2002, 12(4):547-554.

77. Hagemann D, Xiao RP: Dual site phospholamban phosphorylation and its physiological relevance in the heart. Trends in cardiovascular medicine 2002, 12(2):51-56.

78. Narayanan N, Xu A: Phosphorylation and regulation of the $\mathrm{Ca}(2+)$-pumping ATPase in cardiac sarcoplasmic reticulum by calcium/calmodulin-dependent protein kinase. Basic research in cardiology 1997, 92 Suppl 1:25-35.

79. Vangheluwe P, Raeymaekers L, Dode L, Wuytack F: Modulating sarco(endo)plasmic reticulum Ca2+ ATPase 2 (SERCA2) activity: cell biological implications. Cell calcium 2005, 38(3-4):291-302.

80. Zhang Y, Storey KB: Expression of nuclear factor of activated T cells (NFAT) and downstream muscle-specific proteins in ground squirrel skeletal and heart muscle during hibernation. Molecular and cellular biochemistry 2016, 412(1-2):27-40.

\section{Figures}

(a)

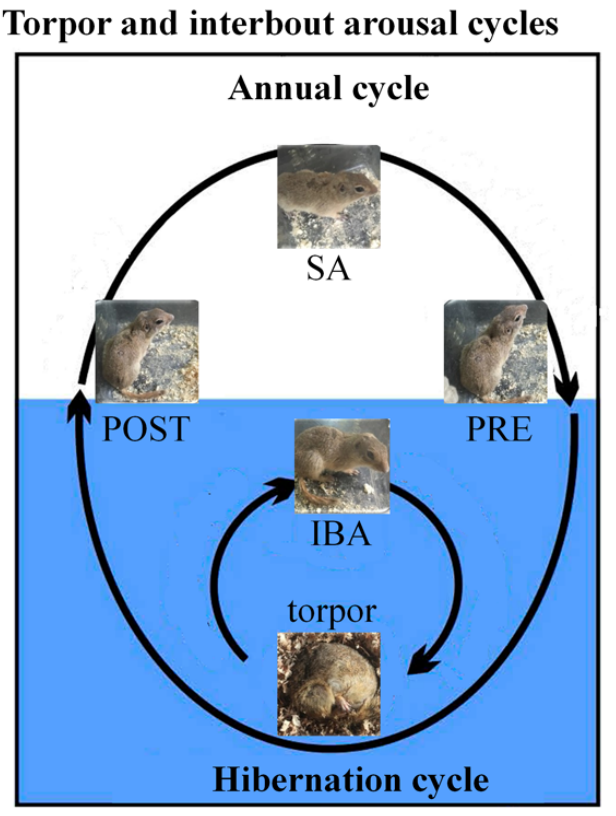

(b)

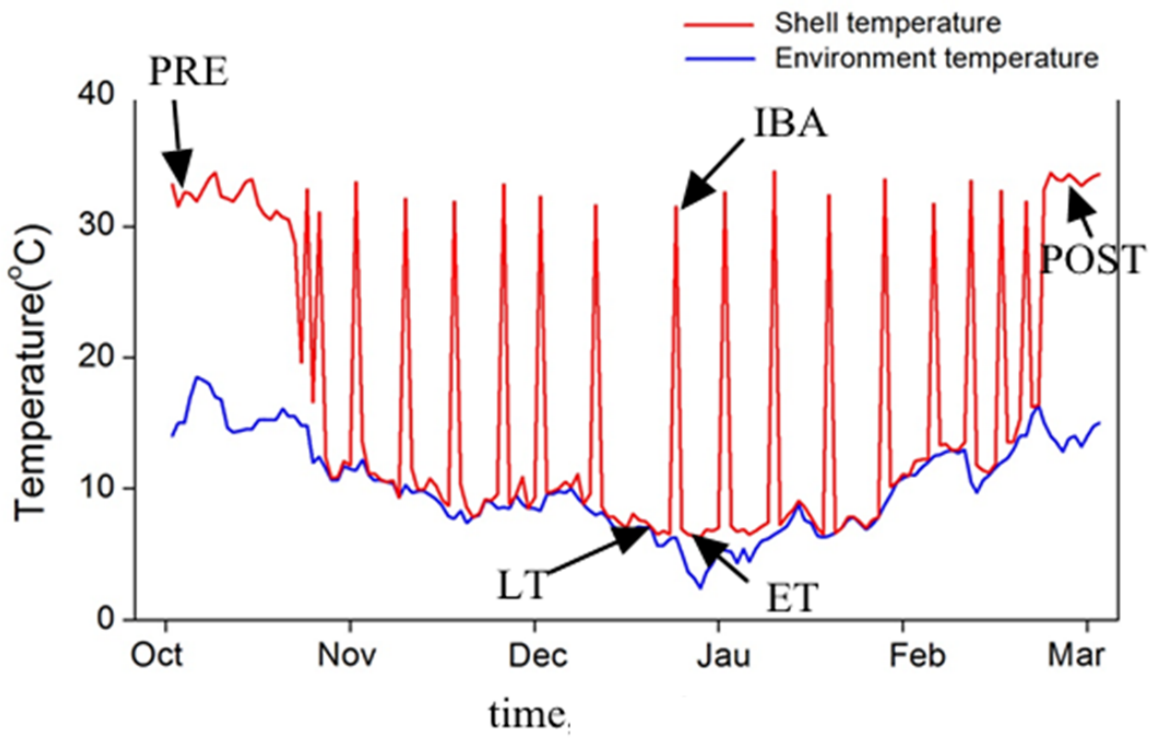

Figure 1

Diagram of hibernating cycle and body temperature in ground squirrels. (a) Animal state of annual and hibernating (blue shading) cycles in ground squirrels. (b) Shell and environmental temperature as a function of time in ground squirrels during hibernation. Red fold line: shell temperature; blue fold line: environmental temperature. SA, summer active; PRE, pre-hibernation; LT, later torpor; IBA, inter-bout arousal; ET, early torpor; POST, post-hibernation. 
(a) SA PRE LT IBA ET POST
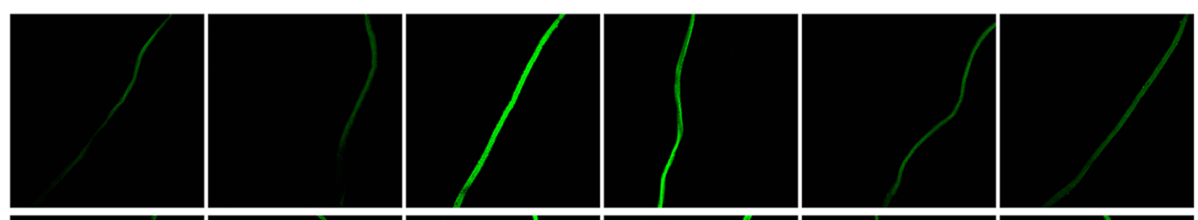

SOL
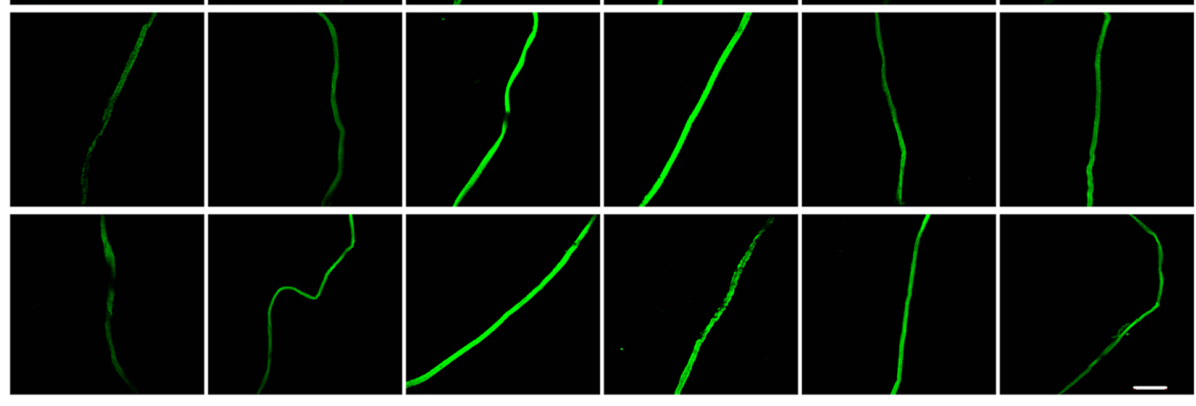

EDL

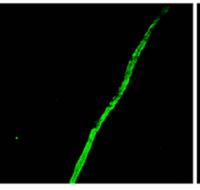

(b)

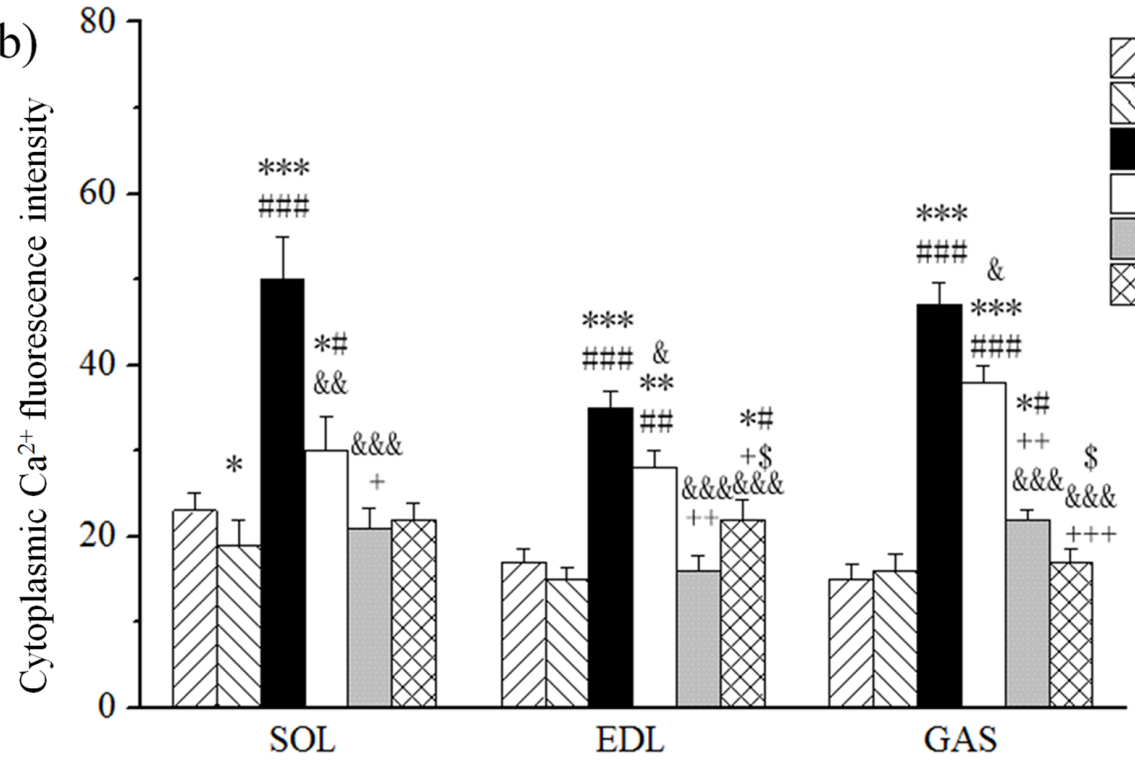

GAS

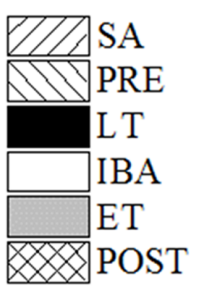

Figure 2

Changes in cytoplasmic Ca2+ fluorescence intensity of single muscle fibers in three different types of muscle during different periods. (a) Representative fluorescence images of single muscle fiber loaded by Fluo-3/AM. Scale bar = $200 \mu \mathrm{m}$. (b) Bar graph depicting changes in mean intensity of cytoplasmic Ca2+ fluorescence. Six muscle fiber cells were analyzed in each sample, eight samples were analyzed in each group. Values are means \pm SE. SOL, soleus muscle; EDL, extensor digitorum longus; GAS, gastrocnemius muscle; SA, summer active group; PRE, pre-

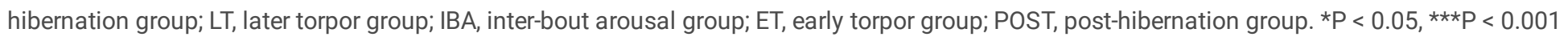
compared with SA; \#P $<0.05$, \#\#P $<0.01$, \#\#\#P $<0.001$ compared with PRE; \&P $<0.05, \& \& P<0.01, \& \& \& P<0.001$ compared with LT; +P $<0.05$, $++\mathrm{P}<0.01,+++\mathrm{P}<0.001$ compared with IBA; $\mathrm{SP}<0.05$ compared with $\mathrm{ET}$. 
(a)

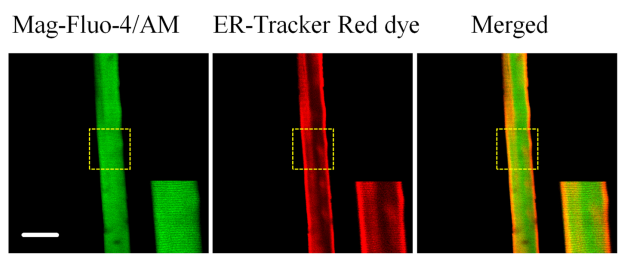

(b) SA

PRE

LT

IBA

ET

POST
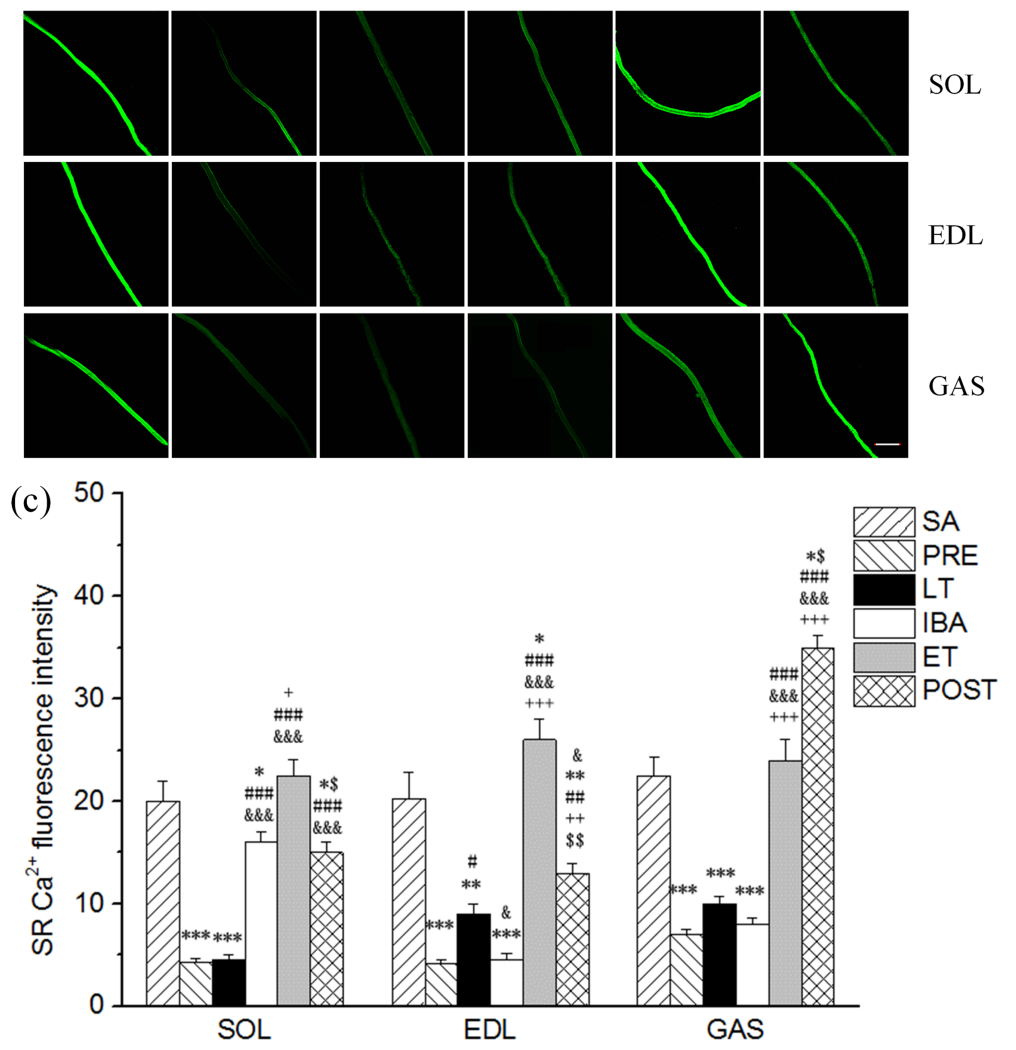

Figure 3

Changes in sarcoplasmic reticulum (SR) Ca2+ fluorescence intensity of single muscle fibers in three different types of muscle during different periods. (a) Representative fluorescence images of single muscle fibers loaded by mag-Fluo-4/AM and ER-Tracker Red dye. Mag-Fluo-4/AM (green) for SR Ca2+, ER-Tracker Red dye (red) for SR. Scale bar $=50 \mu \mathrm{m}$. (b) Representative fluorescence images of fluorescent SR Ca2+ in three muscle types during different periods. Scale bar $=200 \mu \mathrm{m}$. (c) Bar graph depicting changes in mean intensity of SR Ca2+ fluorescence. Six muscle fiber cells were analyzed in each sample, eight samples were analyzed in each group. Values are means $\pm S E$. SOL, soleus muscle; EDL, extensor digitorum longus; GAS, gastrocnemius muscle; SA, summer active group; PRE, pre-hibernation group; LT, later torpor group; IBA, interbout arousal group; ET, early torpor group; POST, post-hibernation group. ${ }^{\mathrm{P}}<0.05$, $* \star * \mathrm{P}<0.001$ compared with $\mathrm{SA}$; \#P $<0.05, \# \# \mathrm{P}<0.01$, \#\#\#P< 0.001 compared with PRE; \&P $<0.05$, \&\&\&P $<0.001$ compared with $\mathrm{LT} ;+\mathrm{P}<0.05,++\mathrm{P}<0.01,+++\mathrm{P}<0.001$ compared with $\mathrm{IBA} ; \$ \mathrm{P}<0.05, \$ \$ \mathrm{P}<$ 0.01 compared with ET. 


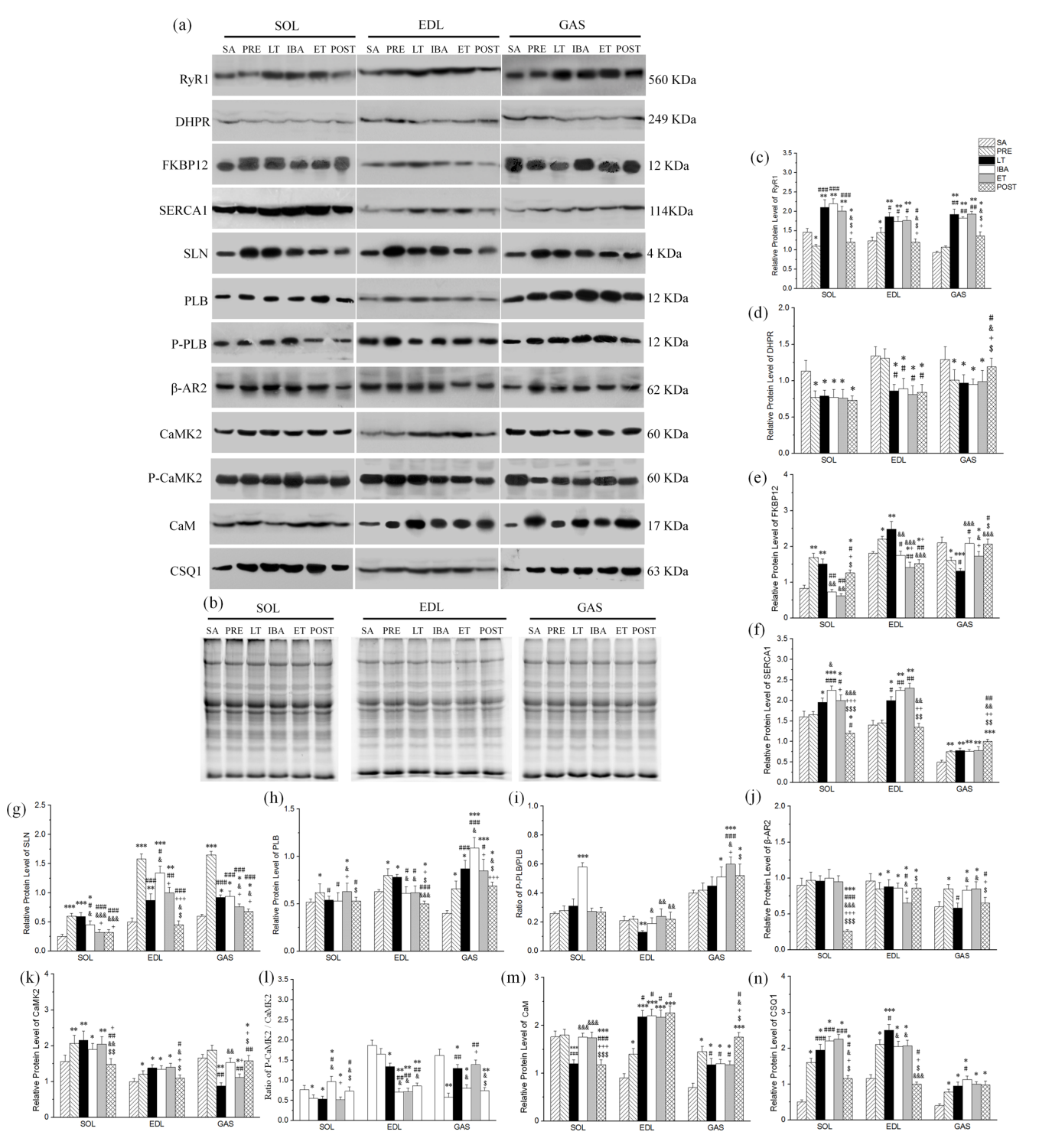

Figure 4

Changes in protein levels of RyR1, DHPR, FKBP12, SERCA1, SLN, PLB, ß-AR2, CaMK2, CaM, CSQ1 and ratios of P-PLB/PLB and P-CaMK2/ CaMK2 in three different types of muscles during different periods. (a) Representative immunoblots of RyR1, DHPR, FKBP12, SERCA1, SLN, PPLB, PLB, $\beta$-AR2, P-CaMK2, CaMK2, CaM and CSQ1 in three different types of muscles during different periods. (b) Representative polyacrylamide gel of total protein. (c) Relative protein expression of RyR1. (d) Relative protein expression of DHPR. (e) Relative protein expression of FKBP12. (f) Relative protein expression of SERCA1. (g) Relative protein expression of SLN. (h) Relative protein expression of PLB. (i) Ratio of P-PLB to PLB. (j) Relative protein expression of $\beta$-AR2. (k) Relative protein expression of CaMK2. (I) Ratio of P- CaMK2 to CaMK2. (m) Relative protein expression of CaM. (n) Relative protein expression of CSQ1. Values are means $\pm S E, n=8$. SOL, soleus muscle; EDL, extensor digitorum longus; GAS, gastrocnemius muscle; SA, summer active group; PRE, pre-hibernation group; LT, later torpor group; IBA, inter-bout arousal

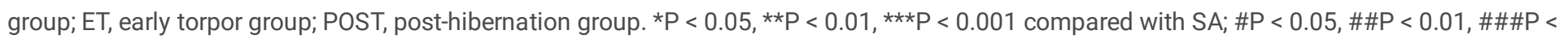
0.001 compared with PRE; \&P $<0.05$, \&\&P $<0.01$, \&\&\&P $<0.001$ compared with LT; $+\mathrm{P}<0.05,++\mathrm{P}<0.01,+++\mathrm{P}<0.001$ compared with IBA; $\$ \mathrm{P}<$ 0.05 ,

$$
P<0.01
$$

\$P $<0.001$ compared with ET. 

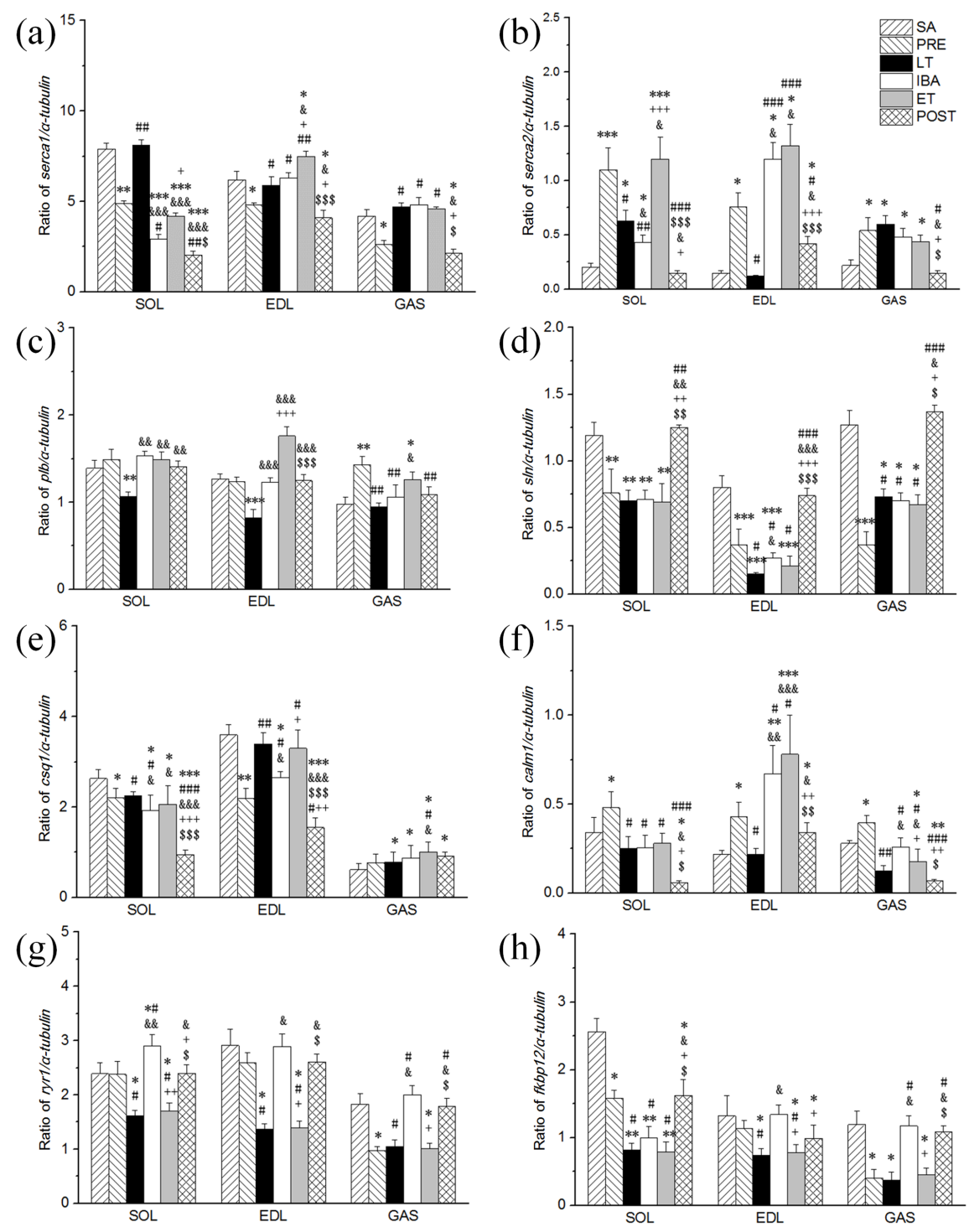

Figure 5

Changes in transcript levels of serca1, serca2, plb, sln, csq1, cam1, ryr1 and fkbp12 in three different types of muscle during different periods. (a) Ratio of serca1/a-tubulin. (b) Ratio of serca2/a-tubulin. (c) Ratio of plb/a-tubulin. (d) Ratio of sln/a-tubulin. (e) Ratio of csq1/a-tubulin. (f) Ratio of cam1/a-tubulin. (g) Ratio of ryr1/a-tubulin. (h) Ratio of fkbp12/a-tubulin. Values are means $\pm \mathrm{SE}, \mathrm{n}=8$. SOL, soleus muscle; EDL, extensor digitorum longus; GAS, gastrocnemius muscle; SA, summer active group; PRE, pre-hibernation group; LT, later torpor group; IBA, inter-bout arousal

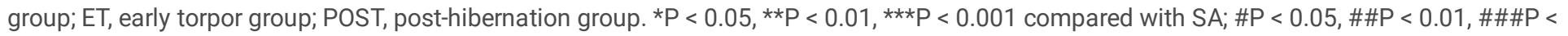
0.001 compared with PRE; \&P $<0.05, \& \& \mathrm{P}<0.01$, \&\&\&P $<0.001$ compared with LT; $+\mathrm{P}<0.05,++\mathrm{P}<0.01,+++\mathrm{P}<0.001$ compared with IBA; $\$$ P $<$ 0.05 ,

$$
P<0.01
$$

\$P $<0.001$ compared with ET. 


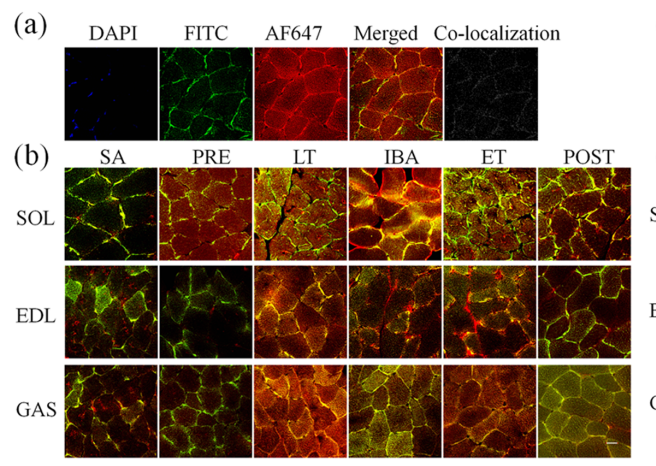

(c)

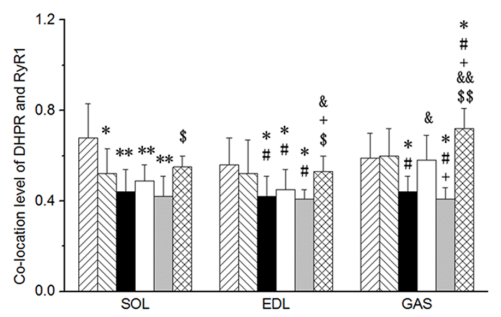

(d)
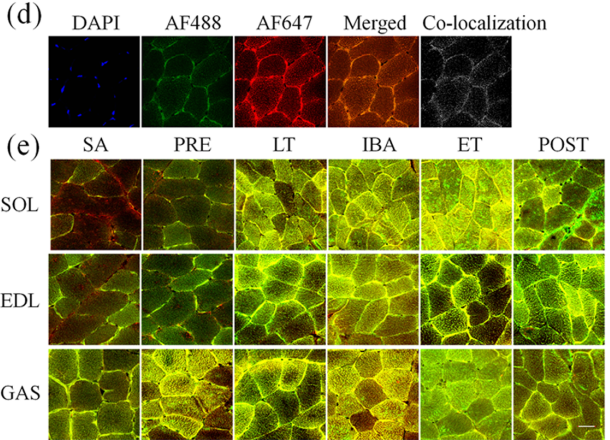

(f)

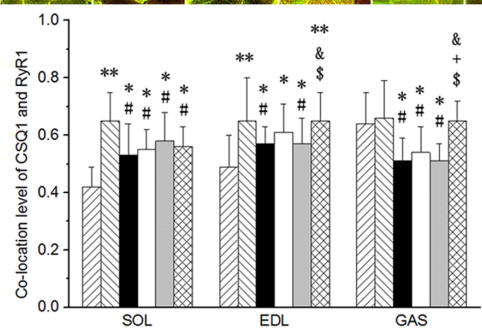

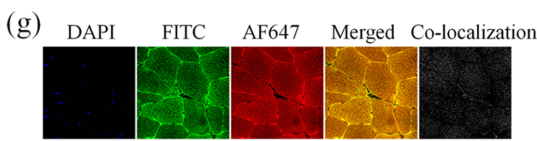

$\begin{array}{lllllll}\text { (h) } & \mathrm{SA} & \mathrm{PRE} & \mathrm{LT} & \mathrm{IBA} & \mathrm{ET} & \text { POST }\end{array}$

SOL

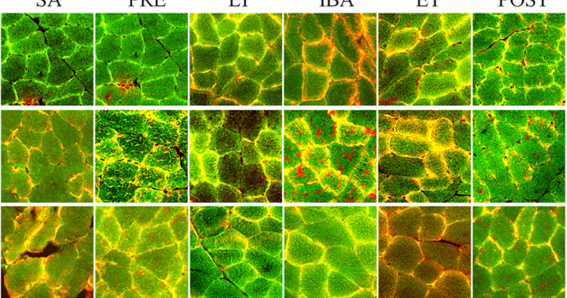

(i)

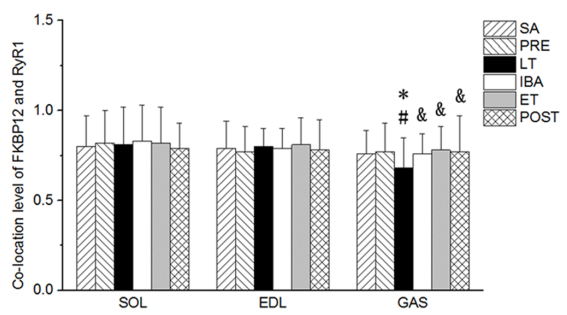

\section{Figure 6}

Protein overlap levels of DHPR/ RyR1, CSQ1/RyR1 and FKBP12/RyR1 in three different types of muscle during different periods. (a) Reticulate subcellular distribution of DHPR and RyR1 fluorescently labeled proteins in three different types of muscle. DAPI (blue) for nuclei, FITC (green) for DHPR, 647 (red) for RyR1, and Merged for overlapping region of two proteins displayed by Image J software. (b) Representative immunofluorescence of protein overlap between DHPR and RyR1 in three different types of muscle during different periods. Scale bar $=20 \mu \mathrm{m}$. (c) Bar graphs representing protein overlap level of DHPR and RyR1. (d) Reticulate subcellular distribution of CSQ1 and RyR1 fluorescently labeled proteins in three different types of muscle. DAPI (blue) for nuclei, AF488 (green) for CSQ1, 647 (red) for RyR1, and Merged for overlapping region of two proteins displayed by Image $\mathrm{J}$ software. (e) Representative immunofluorescence of protein overlap between CSQ1 and RyR1 in three different types of muscle during different periods. Scale bar $=20 \mu \mathrm{m}$. (f) Bar graphs representing protein overlap level of CSQ1 and RyR1.

(g) Reticulate subcellular distribution of FKBP12 and RyR1 fluorescently labeled proteins in three different types of muscle. DAPI (blue) for nuclei, FITC (green) for FKBP12, 647 (red) for RyR1, and Merged for the overlapping region of two proteins displayed by Image $\mathrm{J}$ software. (h) Representative immunofluorescence of protein overlap between FKBP12 and RyR1 in three different types of muscle during different periods. Scale bar $=20 \mu \mathrm{m}$. (i) Bar graphs representing protein overlap level of FKBP12 and RyR1.Values are means $\pm S E, n=8$. SOL, soleus muscle; EDL, extensor digitorum longus; GAS, gastrocnemius muscle; SA, summer active group; PRE, pre-hibernation group; LT, later torpor group; IBA, interbout arousal group; ET, early torpor group; POST, post-hibernation group. ${ }^{*} \mathrm{P}<0.05, \star \star \mathrm{P}<0.01$, ${ }^{\star \star *} \mathrm{P}<0.001$ compared with $\mathrm{SA}$; $\# \mathrm{P}<0.05$, \#\#P< 0.01 , \#\#\#P $<0.001$ compared with PRE; \&P $<0.05$, \&\&P $<0.01$, \&\&\&P $<0.001$ compared with LT; $+\mathrm{P}<0.05,++\mathrm{P}<0.01,+++\mathrm{P}<0.001$ compared with IBA; $\$ \mathrm{P}<0.05$,

$$
P<0.01
$$

$\$ \mathrm{P}<0.001$ compared with ET. 
(a)
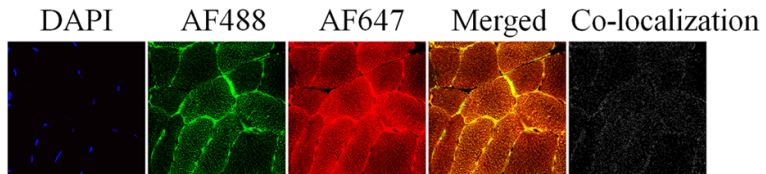

(b)

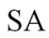

PRE

LT
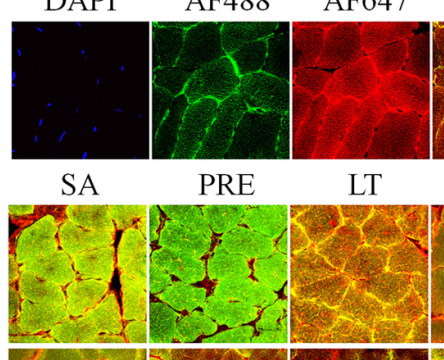

\begin{abstract}
ET
\end{abstract}
SOL

EDL

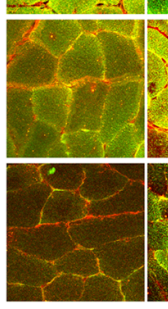

(c)

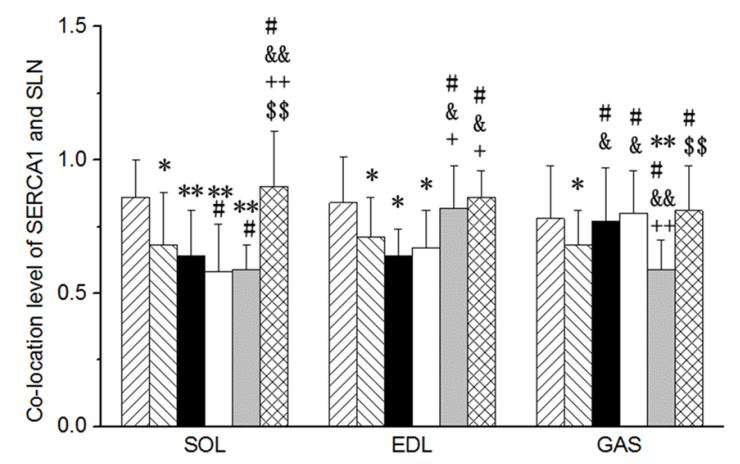

(d)

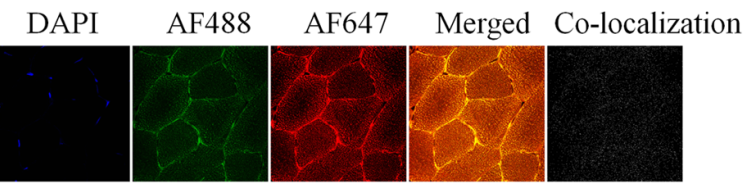

POST

(e)

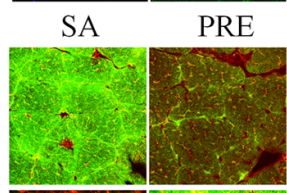

LT

IBA

ET

POST

\section{SOL}

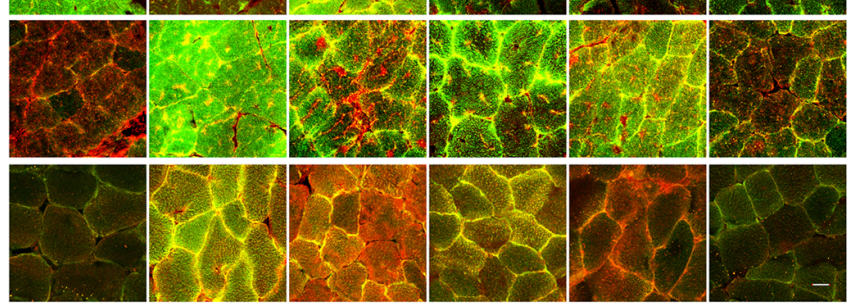

(f)

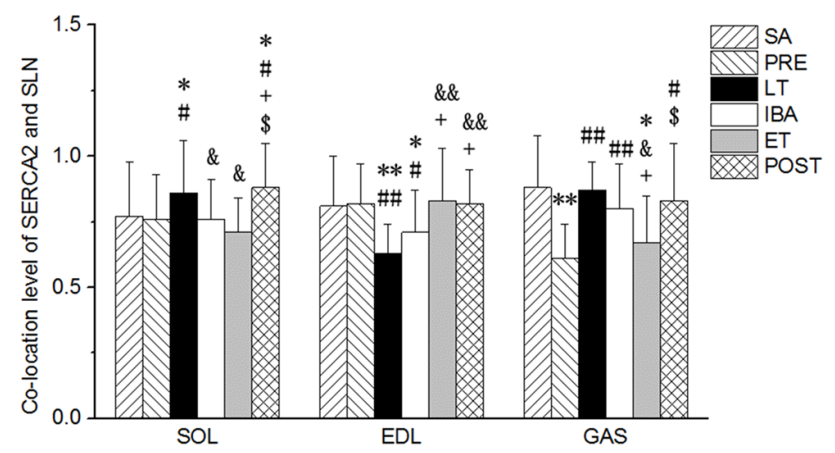

Figure 7

Protein overlap levels of SERCA1/ SLN and SERCA2/SLN in three different types of muscle during different periods. (a) Reticulate subcellular distribution of SERCA1 and SLN fluorescently labeled proteins in three different types of muscle. DAPI (blue) for nuclei, AF488 (green) for SLN, 647 (red) for SERCA1, and Merged for overlapping region of two proteins displayed by Image J software. (b) Representative immunofluorescence of protein overlap between SERCA1 and SLN in three different types of muscle during different periods. Scale bar $=20 \mu \mathrm{m}$. (c) Bar graphs representing protein overlap level of SERCA1 and SLN. (d) Reticulate subcellular distribution of SERCA2 and SLN fluorescently labeled proteins in three different types of muscle. DAPI (blue) for nuclei, AF488 (green) for SLN, 647 (red) for SERCA2, and Merged for overlapping region of two proteins displayed by Image J software. (e) Representative immunofluorescence of protein overlap between SERCA2 and SLN in three different types of muscle during different periods. Scale bar $=20 \mu \mathrm{m}$. (f) Bar graphs representing protein overlap level of SERCA2 and SLN. Values are means $\pm S E, n=8$. SOL, soleus muscle; $E D L$, extensor digitorum longus; GAS, gastrocnemius muscle; $S A$, summer active group; PRE, prehibernation group; LT, later torpor group; IBA, inter-bout arousal group; ET, early torpor group; POST, post-hibernation group. ${ }^{*} P<0.05$, ${ }^{*} P<0.01$ compared with $\mathrm{SA} ; \# \mathrm{P}<0.05$, \#\#P<0.01 compared with $\mathrm{PRE} ; \& \mathrm{P}<0.05$, \&\&P $<0.01$ compared with $\mathrm{LT} ;+\mathrm{P}<0.05,++\mathrm{P}<0.01$ compared with IBA; $\$ \$ P<0.01$ compared with $\mathrm{ET}$. 

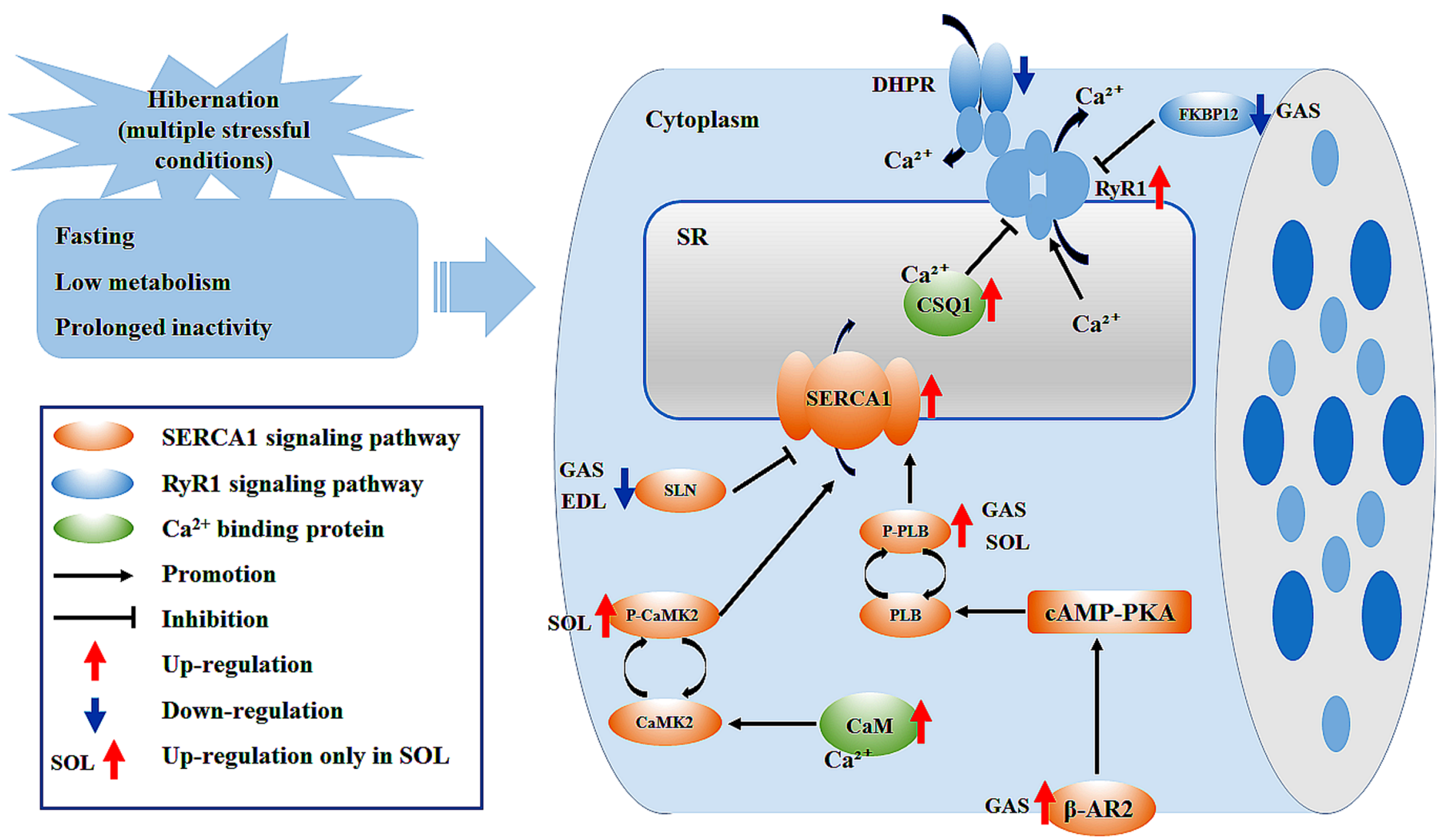

Figure 8

Graphical summary of study. Ca2+, calcium; SR, sarcoplasmic reticulum; DHPR, dihydropyridine receptor; FKBP12, 12 kDa FK506 binding protein; RyR1, ryanodine receptor1; CSQ1, calsequestrin1; SERCA1, sarcoplasmic reticulum calcium ATPase; SLN, sarcolipin; PLB, phospholamban; P-

PLB, phosphorylated phospholamban; CaM, calmodulin; CaMK2, calmodulin kinase2; P-CaMK2, phosphorylated calmodulin kinase2; $\beta$-AR2, betaadrenergic receptor 2; cAMP-PKA, cyclic adenosine monophosphate -protein kinase A signaling pathway.

\section{Supplementary Files}

This is a list of supplementary files associated with this preprint. Click to download.

- SupplementaryMaterial.docx 